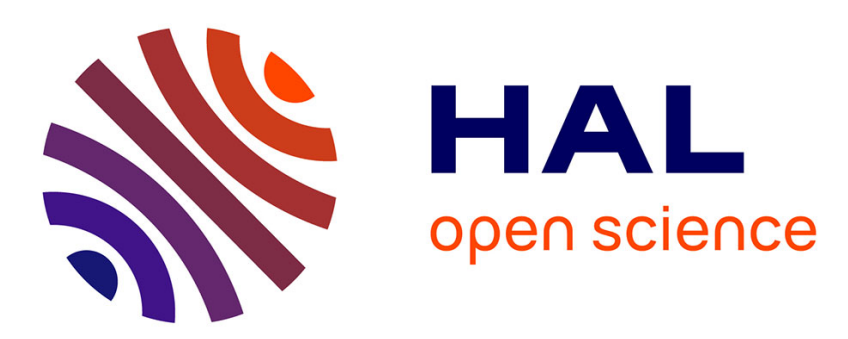

\title{
Technologies et formation: travaux, interrogations, pistes de réflexion dans un champ de recherche éclaté
}

Brigitte Albero

\section{To cite this version:}

Brigitte Albero. Technologies et formation : travaux, interrogations, pistes de réflexion dans un champ de recherche éclaté: Note de synthèse. Savoirs: Revue internationale de recherches en éducation et formation des adultes, 2004, 5 (2), pp.11-69. 10.3917/savo.005.0009 . hal-01713972

\section{HAL Id: hal-01713972 \\ https://hal.science/hal-01713972}

Submitted on 27 Feb 2018

HAL is a multi-disciplinary open access archive for the deposit and dissemination of scientific research documents, whether they are published or not. The documents may come from teaching and research institutions in France or abroad, or from public or private research centers.
L'archive ouverte pluridisciplinaire HAL, est destinée au dépôt et à la diffusion de documents scientifiques de niveau recherche, publiés ou non, émanant des établissements d'enseignement et de recherche français ou étrangers, des laboratoires publics ou privés. 


\title{
TECHNOLOGIES ET FORMATION : TRAVAUX, INTERROGATIONS, PISTES DE RÉFLEXION DANS UN CHAMP DE RECHERCHE ÉCLATÉ
}

\author{
Brigitte Albero
}

\section{L'Harmattan | Savoirs}

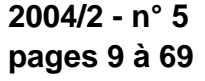

ISSN 1763-4229

Article disponible en ligne à l'adresse:

http://www.cairn.info/revue-savoirs-2004-2-page-9.htm

Pour citer cet article :

Albero Brigitte , «Technologies et formation : travaux, interrogations, pistes de réflexion dans un champ de recherche éclaté »,

Savoirs, 2004/2 n5, p. 9-69. DOI : 10.3917/savo.005.0009

Distribution électronique Cairn.info pour L'Harmattan.

(C) L'Harmattan. Tous droits réservés pour tous pays.

La reproduction ou représentation de cet article, notamment par photocopie, n'est autorisée que dans les limites des conditions générales d'utilisation du site ou, le cas échéant, des conditions générales de la licence souscrite par votre établissement. Toute autre reproduction ou représentation, en tout ou partie, sous quelque forme et de quelque manière que ce soit, est interdite sauf accord préalable et écrit de l'éditeur, en dehors des cas prévus par la législation en vigueur en France. II est précisé que son stockage dans une base de données est également interdit. 
NOTE DE SYNTHÈSE 


\title{
Technologies et formation : travaux, interrogations, pistes de réflexion dans un champ de recherche éclaté ${ }^{1}$
}

\author{
Brigitte ALBERO
}

\author{
Maître de conférences HDR \\ INRP - Groupe inter-universitaire e-pathie $e^{2}$
}

Sciences de l'éducation et technologies n'ont jamais fait bon ménage. Aussi, rédiger une note de synthèse sur un domaine hier conflictuel et soupçonné d'opportunisme, aujourd'hui sinistré par un manque de relève, tient-il de la gageure. La recherche théorique sur ce thème se fait principalement ailleurs, dans des disciplines qui privilégient tantôt l'analyse de l'objet en tant qu'artefact technique, en tant que système sémiotique ou en tant que média de masse, tantôt l'analyse des processus cognitifs que ces technologies stimulent ou inhibent ou encore celle des forces économiques en présence, de leurs incidences sociopolitiques, des nouvelles conditions de travail et des modes d'organisation qu'elles infléchissent.

Trois malentendus récurrents empêchent les sciences de l'éducation de structurer véritablement ce domaine selon leurs propres perspectives : la tendance à confondre pratique et recherche, leurs cadres respectifs de

\footnotetext{
${ }^{1}$ Ce champ de recherche est si vaste et si productif dans de nombreuses disciplines et de nombreux pays qu'il est impossible d'en rendre totalement compte. Cette note de synthèse se donne pour objectif de faire, malgré tout, un point sur le domaine, en privilégiant les travaux réalisés en sciences de l'éducation et en formation des adultes, chaque fois que cela est possible. Les travaux cités le sont à titre d'exemple et ne visent aucunement l'exhaustivité. Je prie donc le lecteur de prendre en compte davantage l'ensemble de l'argumentation que les lacunes qu'il ne manquera pas de relever.

2 http:/ / www.e-pathie.org
} 
références et leurs sémantiques ${ }^{3}$; le soupçon a priori de prise de position techniciste qui évacuerait toute distance critique et enfin la crainte d'une compromission idéologique à l'égard du monde industriel et de ses formes économiques ultra-libérales. Ces malentendus ont plusieurs conséquences : une multiplication de développements de dispositifs et d'outils au détriment de recherches théoriques ; une carence, en dehors de quelques travaux isolés, de formalisation critique méthodique dans le domaine épistémologique et méthodologique; une difficulté à cumuler des résultats hétérogènes, épars, peu visibles et mal (re)connus des autres disciplines qui s'intéressent à des questions proches de ce domaine, mais selon leurs propres perspectives.

Après une période aussi productive qu'hétérogène (années 60-80), les recherches sur les technologies et la formation ont dû se glisser dans les interstices de la section, au hasard des parcours individuels, des besoins ponctuels d'inscription des entités académiques dans une modernité de surface, ou même au hasard (heureux) des vacuités institutionnelles.

Dans un tel contexte, quel peut être l'apport d'une note de synthèse ? Notre propos sera celui, très modeste, d'une tentative d'information auprès d'un public large qui s'intéresse à d'autres objets, en vue de faciliter une possible intercompréhension. Les enjeux économiques, politiques, sociaux, cognitifs, culturels et même biologiques sont tels aujourd'hui qu'ils engagent le sort même des systèmes éducatifs existants. De ce fait, une communauté soucieuse des questions liées à la formation des personnes, quels que soient leur âge et leur situation, ne peut pas ne pas s'intéresser sérieusement à ce sujet.

Cette note propose une approche en trois parties. En premier lieu, des jalons sont posés pour un repérage des recherches qui se font dans d'autres domaines scientifiques et des travaux identifiés en sciences de l'éducation. Dans un deuxième temps, sont avancés des constats et des hypothèses susceptibles de mieux saisir les conditions contemporaines de la recherche dans le domaine de la formation. Enfin, des pistes de réflexion sont proposées pour tenter de

\footnotetext{
${ }^{3}$ Les «spécialistes des technologies en sciences de l'éducation » sont souvent recrutés pour prendre en charge le développement pratique de dispositifs de formation ouverte et/ou à distance. Le manque endémique de personnel et de moyens tend à les transformer en homme ou femme-orchestre et les occupe au point qu'ils n'ont plus aucune possibilité de conduire une activité scientifique de qualité. Ils sont ainsi placés dans une situation paradoxale : le fait même de rendre service à la communauté contribue, en les instrumentalisant, à les marginaliser puisque les critères de sélection et de promotion restent essentiellement centrés sur la recherche. L'intention de conceptualiser se trouve ainsi, systématiquement, supplantée par l'injonction de faire.
} 
situer et définir une recherche qui, sur ces questions, pourrait trouver sa place en sciences de l'éducation.

\section{La recherche sur les technologies et la formation : un champ émietté}

L'analyse des pratiques liées à l'utilisation de supports techniques à visée de formation se structure différemment selon le champ disciplinaire d'inscription des travaux. Elle se centre également sur des questions extrêmement diverses selon le statut accordé aux artefacts eux-mêmes par rapport à d'autres composantes de l'activité : les contenus disciplinaires, les systèmes d'interaction humaine, les conduites, les systèmes symboliques, etc. L'avantage de cette diversité est de produire une littérature abondante et une quantité importante de résultats. Sa contrepartie est son éparpillement, la difficulté à saisir les enjeux des recherches et à relier les résultats les uns aux autres, dans la mesure où chacun adopte des perspectives, des méthodologies et des cadres théoriques différents ${ }^{4}$.

\footnotetext{
${ }^{4}$ Depuis quelques années, diverses tentatives ont été faites pour réunir les acteurs de cette recherche ou pour favoriser la visibilité des travaux. À titre d'exemple : au CNRS, création le 5 octobre 2000 du département STIC (Sciences et technologies de l'information et de la communication) (http://www.cnrs.fr/STIC) avec une ouverture aux problématiques liées à la compréhension des processus instrumentés de connaissance ; en 2002, le Symposium international francophone, intitulé "Les technologies en éducation. Perspectives de recherche et questions vives ", organisé à Paris par G.-L. Baron et E. Bruillard, 31 janvier-1 $1^{\text {er }}$ février, qui réunit des acteurs de la recherche et de l'action politique de différentes instances et dans diverses disciplines ; en 2003, à la MSH de Paris, création du site TEMATICE destiné à réunir et publier sur Internet les travaux de recherche en Sciences humaines et sociales (http://www.tematice.fr) ; le «colloque de prospective », intitulé «Technologies pour l'apprentissage et l'éducation : entre recherche et usages pédagogiques », organisé par la Direction de la technologie (F. Thibault) et la Direction de la recherche (P. Casella), en partenariat avec le CNRS a réuni des chercheurs de différentes disciplines, 25-26 novembre 2003.
} 


\subsection{Grandes orien- tations et lignes de partage} l'analyse des usages sociaux, celle des finalités éthiques et sociopolitiques, l'analyse critique des conditions de production des savoirs.

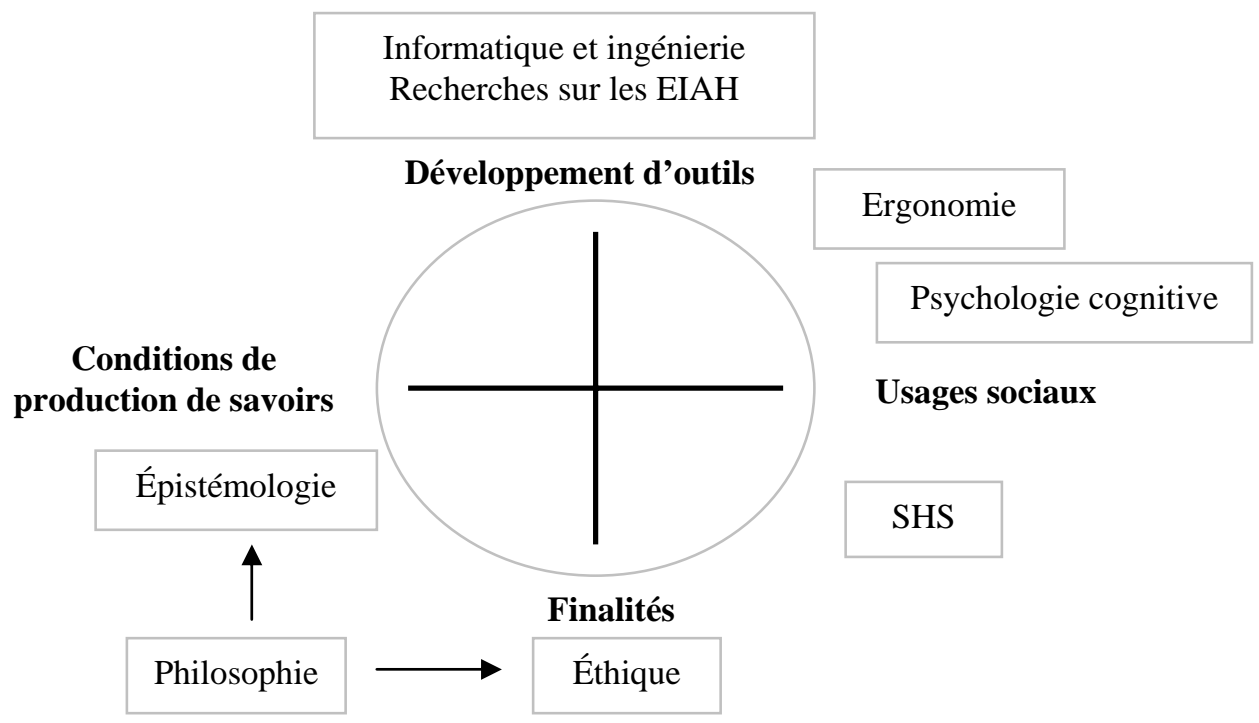

Figure 1: Quatre grandes orientations dans la recherche sur les technologies et les usages à visée de formation des personnes.

Une première orientation s'intéresse aux principes de conception, au développement et à l'évaluation de produits (outils, logiciels, langages, etc.) utiles à l'apprentissage. Les travaux dans ce champ sont représentatifs des secteurs disciplinaires tels que l'informatique, qui, à partir de la décennie 1950

\footnotetext{
${ }^{5}$ Nous entendons «formation» dans son sens le plus large d'action intentionnelle et finalisée, en vue d'obtenir des acquisitions qui modifient la personne. Au-delà de sa dimension ingénierique dans le cadre de l'intervention institutionnelle, cette définition prend en compte les travaux de G. Pineau (1985) sur la structure triadique constitutive de la formation entre éco-, hétéro-, et autoformation.
} 
Note de synthèse - Technologies et formation

(Gardner, 1985, 1993) ont eu tendance à former une interdiscipline (la cybernétique dès 1948, puis l'intelligence artificielle à partir de 1956) en réunissant informaticiens, mathématiciens, psychologues, neurophysiologistes, anthropologues et physiciens. Aujourd'hui, en France, les héritiers de ce courant intéressés par les processus d'apprentissage se regroupent sous la dénomination fédérative des recherches sur les « environnements informatiques pour l'apprentissage humain» (EIAH). Leurs travaux, dont l'approche est le plus souvent expérimentale, s'organisent le plus fréquemment autour d'équipes d'informaticiens, de didacticiens et de psychologues de la cognition ${ }^{6}$. Les résultats de recherche tendent à déboucher sur la proposition de «modèles computationnels de processus didactiques » (Balacheff, 2001) qui dépassent les situations de formation conventionnelles pour s'intéresser plus largement à un éventail d'environnements informatiques ${ }^{7}$ inscrits dans une perspective de transmission de savoirs.

Une deuxième orientation s'intéresse aux usages sociaux des technologies. Ce sont davantage les secteurs disciplinaires regroupés dans la catégorie des sciences humaines et sociales (SHS). Les analyses sont, le plus souvent, le fruit de travaux conduits dans les contextes d'utilisation et auprès des utilisateurs, et portent sur les conditions d'appropriation de l'innovation technique et ses incidences sur les conditions de vie, de travail, de communication et d'apprentissage. Les travaux peuvent être strictement disciplinaires ${ }^{8}$, mais ils peuvent également s'organiser de manière interdisciplinaire dans le cadre de recherches ponctuelles ${ }^{9}$ ou dans le cadre d'une section interdisciplinaire qui s'y prête ${ }^{10}$ plus aisément.

À l'interface de ces deux orientations se développe l'ergonomie qui, en analysant les situations et les activités en contexte, contribue notamment à adapter et faire évoluer les artefacts élaborés par les concepteurs. Les cadres

${ }^{6}$ Par exemple, les cinquante-sept communications recensées par Desmoulins, Marquet, Bouhineau, dans les actes de la conférence EIAH 2003, sont révélatrices des travaux réalisés par ce courant.

${ }^{7}$ Par exemple, une encyclopédie numérique ou un musée virtuel.

${ }^{8}$ Les trente-deux conférences données entre les mois de septembre et octobre 2000 à «L'Université de tous les savoirs» qui étaient centrées sur les «technologies»sont représentatives de cette diversité (UTS, 2002).

9 Par exemple, les Équipes de recherche technologique en éducation (ERTE), lancées en 2002 par le Ministère délégué à la recherche et aux nouvelles technologies.

10 Par exemple, en sciences de l'information et de la communication, les travaux fondateurs de Perriault, 1989 ; Moeglin, 1993. En sciences de l'éducation, ceux de Linard, 1973 ; Jacquinot, 1977, 1985 ; Baron, Bruillard, 1996. Ceux également de Compte, 1985, 1998, à la frontière de ces deux disciplines. 
théoriques et les méthodologies forgées par cette discipline ouvrent des perspectives de recherche pour l'analyse, en « cours d'action » (Theureau, 1992), des situations de formation médiatisée par des artefacts techniques ${ }^{11}$.

Pour sa part, la psychologie cognitive étudie depuis longtemps les détails des opérations mentales et les stratégies individuelles des sujets en situation spécifique d'apprentissage et/ou d'activité professionnelle. Proche de l'ergonomie, elle est souvent partenaire des travaux sur les EIAH ${ }^{12}$.

Une troisième orientation se dégage d'un ensemble de travaux ancrés dans la philosophie ${ }^{13}$. Posant la question des finalités de l'innovation technique, ils portent soit des discours apologétiques ${ }^{14}$ qui considèrent que c'est en devançant les potentialités des techniques que l'être humain construit son avenir ${ }^{15}$, soit des discours plus fortement critiques ${ }^{16}$ qui posent les bases d'une réflexion de type éthique $^{17}$ et/ou politique ${ }^{18}$.

Une quatrième orientation s'inscrit dans une réflexion de type épistémologique qui interroge les cadres de références et les conditions dans lesquelles les savoirs sont produits et diffusés ${ }^{19}$.

${ }_{11}$ Par exemple, les travaux de Leplat, 1997, 2000 ; Clot, 1995, 1999 et Rabardel, 1995, pour les travaux plus spécifiques à l'analyse de l'activité «instrumentée ».

12 Par exemple, Nguyen-Xuan, Grumbach, 1984, 1989 ; Le Ny, Richard, 1986 ; Kayser, 1987 ; Leplat, 1991 ; Nanard, 1994 ; Rabardel, 1995 ; Brien, 1997 ; Tricot, Rouet, 1998 ; Legros, Crinon, Georget, 2000 ; Legros, Crinon, 2002.

${ }^{13}$ Sur la base des travaux fondateurs notamment de Heidegger, 1954 ; Habermas, 1968 ; Simondon, 1958, 1989 ; ou encore Ducassé, 1958 ; Hottois, 1984, 2001.

${ }^{14}$ Par exemple, Lévy, 1987, 1990, 2000.

15 Par exemple, de Rosnay, 1991, 1995, 2001.

16 Par exemple, Wolton, 2000 ; Finkielkraut, Soriano, 2001 ; Virilio, 1998 ; Stiegler, 2004a/b. Aux États-Unis, les travaux de Noble, 2001, sont révélateurs de ce courant de pensée.

${ }_{17}$ Par exemple, en philosophie, Jonas, 1979, 1993. En informatique, Aiken et Eptstein, 2000 ; Schneiderman, 1999. En sciences de l'éducation en France, Linard, 2003.

18 Par exemple, en France : Ellul, 1954, 1977 ; Roqueplo, 1983. Aux États-Unis, Marcuse, 1964 ; Scove, 1995 ; Feenberg, 1999.

19 Par exemple, en sciences de l'information et de la communication, Stiegler, 1994 ; Sfez, 2002. En sociologie des sciences, Latour, 1999. En sciences de l'éducation, Linard, 1989 ; Clergue, 1997. Pour Monique Linard, le problème de l'intégration des technologies dans l'éducation et la formation est avant tout celui des fondements théoriques de la médiation dans les dispositifs ou plutôt celui de l'indifférence à ces fondements, entraînée par la domination des aspects techniques et gestionnaires sur les aspects cognitifs, pédagogiques et sociaux. 
À l'intérieur de ces orientations principales, quatre perspectives dessinent d'autres lignes de partage.

Une première perspective, de type diachronique, oriente des travaux qui s'organisent selon une chronologie déterminée par le stade de développement des objets techniques étudiés et des pratiques de formation qui leur sont attachées. Chaque innovation technique entrainant son lot de curiosités, d'observations, d'études, d'analyses.

Une deuxième perspective, de type strictement disciplinaire, se dessine avec des travaux qui définissent l'angle d'analyse adopté selon la discipline de rattachement du chercheur. Ainsi un même artefact sera-t-il analysé en tant qu'objet technique, ou selon les processus cognitifs qu'il suscite, les codes sémiotiques qui le caractérisent, les systèmes d'interaction et de communication qu'il produit, les pratiques didactiques et pédagogiques qui l'accompagnent.

Une troisième perspective, de type théorique, regroupe des travaux qui étayent leurs analyses sur des cadres conceptuels référencés dans des domaines disciplinaires précis (philosophie, psychologie, sociologie, économie, informatique, etc.).

Une quatrième perspective, de type pragmatique, comporte des travaux qui recouvrent, explicitement ou non, deux types de finalités conceptualisées par J.M. Barbier (2001) : l'intelligibilité des phénomènes étudiés et/ou l'optimisation des actions, qu'il s'agisse de recherche-action, de recherche-action-formation ${ }^{20}$ ou encore de recherche-développement.

Ces quatre grandes orientations et quatre perspectives ne sont pas exclusives les unes des autres, mais le fait même de les dégager peut permettre de situer les travaux de recherche dans un foisonnement de production de plus en plus dense. En effet, le développement ultra-rapide et la banalisation des technologies dans tous les domaines de la vie courante modifient à ce point les conditions d'existence que de nombreux secteurs scientifiques s'y intéressent de plus en plus. Le secteur de la formation, perçu, depuis quelques années, comme une niche économique potentielle, génère un intérêt accru depuis l'avènement d'Internet, surtout dans les secteurs de la formation continue des salariés et de l'enseignement supérieur.

20 Cette approche est particulièrement développée par la recherche belge (Charlier, Peraya, 2003). 
Savoirs, 5, 2004

\subsection{En sciences de \\ l'éducation, une recherche qui tend à s'inscrire dans une perspective diachronique}

Durant le demi-siècle qui vient de s'écouler, les «machines à enseigner ${ }^{21}$ » (Skinner, 1958), «à communiquer » (Schaeffer, 1970), «à représenter » (Linard, 1987), «à penser» (Arsac, 1987), tout comme les usages qu'elles contribuent à générer ne cessent d'être étudiées par les chercheurs intéressés aux questions de la formation des personnes. En sciences de l'éducation, cette recherche est fortement reliée aux préoccupations des acteurs éducatifs et politiques, elle est donc souvent conduite en liaison avec les terrains de la formation et avec les initiatives des pouvoirs publics. Cette particularité peut expliquer cette prépondérance de la perspective diachronique et l'emploi d'une terminologie qui tend à suivre l'évolution des dénominations des outils dans le temps. Cette situation particulière crée régulièrement un afflux de productions et une activité de réseau $(\mathrm{x})$ qui peut prendre, à certains moments, toute l'apparence d'une structuration de champ. Cette production par vagues successives (Baron, Bruillard, 1996 ; Baron, 2001) a le mérite de créer une dynamique et une mémoire de l'évolution des techniques dans le domaine de la formation. Elle rencontre cependant la limite d'une durée liée à la rapide obsolescence des outils ou à leur transformation radicale. Il en résulte une difficulté à capitaliser les apports empiriques et conceptuels de chacune de ces vagues, dont la force tend à être amoindrie, voire annulée, par la vigueur que confère la nouveauté à la suivante 22 .

Par ailleurs, le repérage des datations des périodes successives n'est guère aisé, car trois moments se succèdent, créant des décalages temporels : celui des recherches techniques sur un nouveau support; celui de son entrée dans le social et notamment dans le monde de la formation ${ }^{23}$ (expérimentation,

\footnotetext{
21 Traduit de l'américain teaching machines, cette expression, critiquée par M. Montmollin (1965), a été reprise et analysée par P. Moeglin (1993) dans une contribution qui donne à ce terme une dimension paradigmatique, révélatrice des tentatives de mécanisation et d'industrialisation des processus éducatifs depuis le XIXe siècle.

${ }^{22}$ La double temporalité du développement technologique et de ses usages sociaux et celle de la recherche placent le chercheur dans une position paradoxale : soit il tente de suivre le rythme technologique et social et ses travaux peuvent être jugés opportunistes et superficiels ; soit il suit une direction théorique de recherche et ses travaux sont jugés anachroniques ou abscons.

${ }^{23}$ La datation de ce moment intermédiaire demanderait des recherches précises, car il est à remarquer que les supports techniques nouveaux sont généralisés dans les secteurs de la formation des adultes, bien plus tôt que dans les secteurs scolaires.
} 
développement à partir d'incitations et de soutiens des pouvoirs publics, généralisation toujours problématique); celui des recherches conduites sur son utilisation en formation et d'une communication publique de résultats. Entre les deux moments extrêmes, il a pu s'écouler une vingtaine d'années. Pour garder une cohérence avec les datations repérées par d'autres chercheurs, nous avons fait le choix, dans cette note, de prendre comme point de repère le moment intermédiaire d'entrée d'un support technique dans le monde de la formation. Cela permettra de remarquer que les datations des travaux de recherche cités s'échelonnent sur une temporalité qui dépasse l'entrée du support technique suivant, créant des chevauchements qu'il serait intéressant d'analyser de manière précise.

Les années soixante ${ }^{24}$ marquent le début d'un intérêt systématique et organisé pour ce qui est alors appréhendé comme un «auxiliaire» de l'enseignement. À partir de cette période, il est possible de repérer cinq vagues successives au cours desquelles la désignation par le moyen technique tend à être associée à une désignation par l'usage formatif auquel celui-ci est attaché. Indépendamment de quelques rares travaux à caractère historique ${ }^{25}$ ou liés à une discipline précise ${ }^{26}$, il est ainsi possible de distinguer :

- À partir des années 1960, période des «médias audiovisuels » et de la " télévision éducative », associée au « télé-enseignement».

- À partir des années 1970, entrée de l'informatique avec le développement de la micro-informatique, associée à l' " enseignement programmé », l' « enseignement assisté par ordinateur » (EAO) et l' « enseignement à distance » (EAD).

- Les années 1980 voient apparaitre le «multimédia», les "nouvelles technologies » et les «technologies de l'éducation » désignées, en fin de période, sous de nombreux acronymes (NTE, NTF, NTIC), associées à l' « enseignement intelligemment assisté par ordinateur » (EIAO) et à la « formation à distance » (FAD).

${ }^{24}$ Dans le champ social, c'est la décennie de l'expansion massive des médias de communication. Dans le champ académique, c'est celui de la création des sciences de l'éducation comme discipline (1967). Ce double mouvement peut expliquer l'intérêt accru pour des médias susceptibles de relayer le travail éducatif de l'institution et la production plus systématique de travaux de recherche.

${ }_{25}$ Par exemple, Béranger Sert, 1981 ; Rubenach, 1990.

${ }^{26}$ Par exemple, en arts, Sultan, Vilatte, 1998 ; Sultan, 2000. En histoire, Poinssac-Niel, 1975. En informatique, Bruillard, 1997. En langues, Compte, 1984, 1993 ; BucherPoteaux, 1997 ; Chanier, Pothier, 1998 ; Mangenot, 1998. En sociologie, Blandin, 2002. 
- Les années 1990 voient un intérêt accru pour l' «hypermédia », les «technologies de l'information et de la communication » (TIC), "TIC pour l'éducation » (TICE), et «technologies numériques », associées à la « formation ouverte et à distance » (FOAD).

- À partir des années 2000, il est possible de voir s'amorcer une nouvelle vague autour du développement des « plates-formes numériques » et de la dénomination "espaces numériques » qui semble être associée au développement des «espaces numériques de travail» (ENT), et notamment dans le secteur de l'enseignement supérieur, à celui des «campus numériques ».

1.2.1. Années $1960:$ Médias audiovisuels, télévision éducative et télée-enseignement
Depuis les travaux sur l'image fixe et animée ${ }^{27}$ jusqu'à la télévision éducative, cette période, extrêmement fructueuse, voire prolifique, pose toutes les bases de la réflexion éducative sur les médias. Les travaux sur la télévision scolaire et le film pédagogique en particulier apportent nombre d'analyses qui rendent explicites les limites 28 de la télévision dans les contextes institutionnels de la formation et celles d'une «société pédagogique» selon l'expression de J. Beillerot (1982) où le média, détourné de sa fonction première - sous couvert d'intentions louables - perd son caractère spécifique et son potentiel médiateur (Compte ${ }^{29}$, 1984). Les travaux menés sur le grand domaine inexploré qu'est la télévision grand public ${ }^{30}$ conduisent à prendre en compte les formes d'apprentissage que les personnes

27 Par exemple, Dieuzeide, 1965, 1974 ; La Borderie, 1972 ; Linard, 1975 ; Oppenheim, 1977 ; Baptiste, Bélisle, 1978.

${ }^{28}$ Par exemple, Schaeffer, 1980.

${ }^{29}$ À partir d'une approche cognitive du média (Neisser, 1976 ; Salomon, 1979, 1981), ce chercheur a réalisé de nombreux travaux qui débouchent sur la démonstration de l'existence d'une «rhétorique télévisuelle» (Compte, 1985a/b) dans l'écriture télévisuelle nationale (ibid., 1993) et internationale (ibid., 1985a, 1999). En facilitant la compréhension (ibid., 1985a, 1995), ce système de régularités lié à des «intentionnalités » précises, modifie les représentations des spectateurs (ibid., 1985a, 1993, 2001) et joue un rôle de médiation dans les apprentissages (ibid., 1985a, 2001, 2003). La puissance de cette médiation «technique », très utile dans un contexte didactique (ibid., 1984, 1995), tient au respect des principes de communication qui équilibrent trois composantes de l'échange interpersonnel : le verbal, le non-verbal et le contextuel (ibid., 1985a, 1995).

30 Par exemple, Flageul, 1974 ; Souchon, Poulet, 1976 ; Sultan, Satre, 1981 ; Mariet, 1989. 
réalisent en dehors de l'institution éducative ${ }^{31}$. Sous la double influence intellectuelle de C. Metz et de M. Tardy, G. Jacquinot $(1977$; 1985) ouvre la voie d'une sémiologie du média audiovisuel (à la fois télévisuel et filmique) en tant que support «didaxique». Tout comme chez M. Egly (1981; 1984), une posture critique oriente les travaux de ce chercheur : à l'égard d'une institution éducative "prégnante» face à la "pertinence mass-médiatique» (Jacquinot, 1977, p. 30) ; à l'égard des "pédagogues » à ce point «aveuglés » par «les problèmes de contenu » qu'ils ne voient pas - ou ne savent ${ }^{32}$ pas - que le média n'est pas seulement un véhicule d'information, mais peut aussi se constituer en « instrument de pensée » (ibid., p. 20). Pour ce chercheur le problème principal de l'intégration des technologies est la question des choix pédagogiques et celle des finalités éducatives poursuivies.

Parallèlement, des travaux rapportent des expériences ${ }^{33}$ pédagogiques inédites au cours desquelles les élèves participent à la réalisation télévisée d'émissions destinées à leur propre formation. Ces travaux présentent ces expériences comme des situations alternatives à la situation de simple réception d'émissions éducatives diversement exploitées par les enseignants ${ }^{34}$ surtout dans des fonctions illustratives (Jacquinot, 1977 ; Compte ${ }^{35}$, 1985b). Dans cette même perspective, la banalisation, puis la miniaturisation des matériels audiovisuels, permettent de les introduire dans la classe. L'enregistrement par vidéo de l'activité des individus et le visionnement instantané qu'il rend possible conduisent à un ensemble de travaux sur l'autoscopie en tant qu'outil de formation. La «vidéo-formation» est ainsi utilisée pour permettre la construction des compétences professionnelles, notamment chez les

31 Par exemple, à la suite de G. Friedmann (1966), L. Porcher (1974) utilise la notion d' «école parallèle» en s'intéressant aux pratiques d'autodidaxie que suscite le média télévisuel.

32 G. Jacquinot (1977) épingle « la pauvreté de notre savoir sur ce que sont ces messages (audiovisuels) et comment ils fonctionnent » (ibid., p. 14).

33 G. Berger, E. Brunswick, G. Jacquinot ont contribué à impulser l'expérience du Collège Louis Lumière de Marly-le-Roi (1963-1975) où ont été expérimentées de nombreuses situations pédagogiques, notamment celles qui étaient réalisées autour d'un circuit fermé de télévision comportant une régie. Voir Jacquinot, 1985.

34 Par exemple, M. Tardy (1962 ; 1966 ; 1976) met en valeur l'intérêt du travail pédagogique direct avec le support visuel par rapport à la seule réception d'une émission.

35 C. Compte (1985b ; 1993) dégage trois fonctions pédagogiques (document illustrateur, déclencheur, moteur) respectivement reliées à trois fonctions psychologiques (modélisation, médiation, facilitation) qui permettent de faire avancer la réflexion sur ce sujet. 
enseignants et les formateurs ${ }^{36}$. Des expérimentations ${ }^{37}$ mettent en évidence les dimensions sociales et psychologiques, variables selon les structures de personnalité, de la perception individuelle de l'image de soi, à partir du moment où elle est objectivée et publiquement exposée à l'écran pour analyse et évaluation. Ces expériences montrent en quoi l'autoscopie touchant au sentiment d'identité des personnes peut avoir des effets très divers, positifs et négatifs, sur les attitudes et les conduites d'apprentissage et doit être pratiquée avec précaution ${ }^{38}$.

À la fin de cette période commencent à apparaître des travaux critiques sur les effets négatifs de la télévision auprès des publics jeunes ${ }^{39}$. D'autres font un bilan sans concession de cette période ${ }^{40}$ ou font le point sur des expériences conduites en France ${ }^{41}$ et à l'étranger ${ }^{42}$. Quelques travaux, plus rares, portent davantage sur les usages ${ }^{43}$, indépendamment des technologies qui les suscitent.

C'est durant cette période que se développe la notion de "téléenseignement», expression qui sous-tend l'idée selon laquelle l'activité d'enseignement peut être télé-portée par des moyens techniques de diffusion et/ou de reproduction. Si l'on peut y voir un prolongement modernisé - au moins dans les termes - des plus anciens «cours par correspondance »

\footnotetext{
36 Par exemple, Fauquet, Strasvogel, 1972 ; Freiche, 1972 ; Mottet, 1976-1979, 1997; Compte, 1993.

${ }^{37}$ Par exemple, Linard, 1973 ; Linard, Prax, 1984.

38 Par exemple, M. Linard (1973) met en valeur les effets du retour d'information apporté par l'image autoscopique sur les processus liés aux activités d'enseigner et d'apprendre. Elle met en valeur un certain nombre d'implications de l'autoscopie pour l'image de soi et d'effets sur les attitudes et les comportements des individus en situation de formation, notamment : «un rôle (...) de catalyseur d'affect» (ibid., p. 167); « les réactions à l'image vidéoscopique de soi sont (...) différentes (...) selon les personnalités (...) les rôles sociaux » (ibid., p. 168) ; les groupes prennent «plus vite conscience de leurs difficultés spécifiques, les localisent et les traitent plus efficacement accédant ainsi plus rapidement à de meilleurs résultats » (ibid., p. 172-173); cela conduit à « un effet d'accélération » (ibid., p. 173) ; l'attitude des sujets liée à leur personnalité et à leur histoire est "une variable essentielle qui interfère avec les effets propres au moyen technique » (ibid., p. 175).

39 Par exemple, Chalvon, Corset, Souchon, 1979 ; Azemard, 1980 ; Lurcat, 1981 et 1984 ; Egly, 1981 ; Isambert-Jamati, 1984.

40 Par exemple, Delaville, 1986.

${ }^{41}$ Par exemple, Bireaud, 1979 et 1982.

42 Par exemple, Chaniac, 1975.

43 Par exemple, M. Tardy (1974) analyse la pratique du travail indépendant dans le centre d'autodocumentation du Collège Louis Lumière de Marly-le-Roi.
} 
(Glikman, 2002), ceux-ci continuent de répondre à l'idée selon laquelle les moyens techniques permettent de diffuser des enseignements auprès de publics éloignés des lieux de la formation, sans pour autant déboucher sur des changements structurels dans les pratiques de formation.

\subsubsection{Années 1970 :} Informatique et enseignement programmé, enseignement assisté par ordinateur (EAO), enseignement à distance $(\mathrm{E} A D)$

Impulsé par les travaux du psychologue américain B. Skinner (1954, 1958, 1968) à partir d'une approche béhavioriste de l'apprentissage, l'enseignement programmé est expérimenté depuis les décennies 50-60, principalement par des psychologues $^{44}$, mais ce n'est que durant les années 1970 qu'il monte en puissance dans le champ des pratiques éducatives. Le but est d'optimiser les capacités de répétition et de renforcement offertes par l'ordinateur. Par rapport à d'autres moyens techniques - notamment la télévision éducative -, celui-ci apporte pour la première fois une possibilité d'individualisation de l'enseignement. La notion d' « auto-instruction » apparait (Montmollin, 1965).

De nombreux travaux se font dans les laboratoires sur le développement de langages auteurs et de didacticiens dont de nombreuses traces n'ont pas été publiées, quelques travaux sont destinés aux praticiens ${ }^{45}$, plus rare est la production réflexive et théorique ${ }^{46}$ au plan didactique et pédagogique, voire la production critique ${ }^{47}$. Parallèlement, les recherches en intelligence artificielle, réalisées en grande partie aux États-Unis (Minsky, 1968, 1985 ; Simon, 1969, 1974 ; Schank, Abelson, 1977), conduisent à des travaux sur les systèmes experts plus largement expérimentés dans les domaines scientifiques.

Durant les décennies 70-80, quatre événements contribuent, en France, à augmenter l'intérêt pour l'informatique et à développer des travaux dans ce domaine :

- Une avancée technico-industrielle avec l'arrivée de la microinformatique pour le grand public.

44 Par exemple, en psychologie, Montmollin, 1965, 1971 ; Decote, 1967.

45 Par exemple, Gavini, 1965 ; Don, 1988.

46 Par exemple, en linguistique, Demaizière, 1986. En sciences de l'information et de la communication, Mucchielli, 1987. En science de l'éducation francophone, Depover, 1987.

${ }^{47}$ Par exemple, Lamouroux, More, 1987. 
- Un événement scientifique avec la publication de l'ouvrage de S. Papert (1981) qui constitue un moment charnière dans la manière d'aborder les technologies dans le cadre de la formation, puisque avec le système LOGO, il met à disposition et exploite les possibilités qu'offre l'ordinateur de favoriser les apprentissages interactifs de type manipulatoire et exploratoire.

- Deux décisions politiques avec l'entrée de l'informatique comme discipline scolaire dans le secondaire ${ }^{48}$ (1970) et, plus tard, le Plan informatique pour tous (IPT, 1985). La dotation massive d'un parc informatique dans les établissements scolaires permet de faire prendre conscience des potentiels et des limites de l'outil informatique pour l'apprentissage.

Cette création disciplinaire dans le champ des pratiques scolaires conduit parallèlement à une plus grande autonomisation, dans le champ de la recherche, des axes « informatique » et « didactique de la technologie »49.

En prenant appui sur les travaux béhavioristes en psychologie, les recherches conduites autour des thématiques de l' «enseignement programmé » et de l' "enseignement assisté par ordinateur»(EAO) tendent à techniciser davantage le rapport à l'apprentissage, en focalisant les situations de formation exclusivement sur l'activité d'enseignement. Cette approche est également à l'œuvre dans le développement des dispositifs d'enseignement à distance (EAD). Si ce type de centration a permis d'explorer les potentiels des moyens techniques, il a aussi contribué à en montrer - parfois de manière caricaturale les limites. Certains travaux font le point sur ces questions ${ }^{50}$. Dans ce contexte, certains chercheurs s'interrogent également sur les conditions de formation des enseignants et des formateurs ${ }^{51}$.

\footnotetext{
48 Par exemple, Audouin, 1971 ; Baron, 1989 ; Grandbastien, 1989.

49 Par exemple, Martinand, 1994, 1995 ; Lebeaume, Martinand \& al., 1998 ; Lebeaume, 2000.

50 Par exemple, En France : Lehnisch, 1980. En Angleterre : Kaye A., Rumble G., 1981 ; Kaye, 1988.

51 Par exemple, Altet, Britten, 1983 ; Blandin, 1990 ; Baron, Baudé, 1992 ; Annoot, 1996.
} 
Note de synthèse - Technologies et formation

1.2.3. Années 1980 :

Multimédia, nouvelles

technologies, enseignement

intelligemment assisté par

ordinateur (EIAO) et

formation à distance (FAD)
Avec l'apparition de nouveaux moyens techniques et l'évolution des pratiques, les qualificatifs et acronymes se multiplient, laissant apparaître des centrations, des cultures ou tout au moins des sensibilités différentes ${ }^{52}$. Par exemple, en sciences de l'éducation, les débats autour des termes «technologie de l'éducation» et «technologie éducative » sont révélateurs du climat de cette période.

Les termes «technologie de l'éducation» et "technologie éducative», proches de l'anglais instructional technology, respectivement introduits en France dans les années 1970 par E. Brunswic (1970) et H. Dieuzeide (1970) créent, pour un temps, un mouvement de fédération qui ne va pas sans confrontations. Il faut cependant attendre les années 1980 pour qu'une réflexion faisant la synthèse des débats autour de ces notions soit produite au Canada (Scholer, 1983). Durant cette période, une partie des discussions porte sur la tension entre l'importance accordée par la recherche à l'outil et l'importance accordée à l'action. Les tenants de l'acception «technologie de l'éducation» mettent en valeur les caractéristiques des outils mis au service de l'offre de formation; les tenants de l'acception « technologie éducative » signifient par cette nuance qu'ils s'intéressent davantage au savoir-faire pédagogique lié à l'utilisation des outils techniques. Une troisième tendance se dégage qui consiste à travailler sur les apprentissages auxquels peuvent conduire les médias. Le double passage de la conception de l'artefact comme outil d'un enseignement institué à la conception de l'artefact comme média destiné à un public plus large, et de la centration sur l'offre de formation instituée (par exemple, l'enseignement) à la prise en compte d'un public non captif (par exemple, le téléspectateur) tend à placer ce type de travaux en marge du champ de l'éducation et à le renvoyer vers le champ de l'information et de la communication. Pourtant cette troisième voie/x aurait pu s'avérer extrêmement féconde si elle avait été pleinement assumée, c'est-à-dire conceptualisée et développée, à la frontière de ces deux champs académiques.

Parallèlement à ces débats, des recherches ponctuelles se ramifient selon les types de supports (télématique ${ }^{53}$, vidéodisque ${ }^{54}$, utilisation des satellites ${ }^{55}$ pour la

52 Entre les Nouvelles technologies pour l'éducation (NTE), pour la formation (NTF) et les Nouvelles technologies de l'information et de la communication (NTIC) le champ de pratiques concerné n'est pas le même (secteur scolaire pour le premier; secteur de la formation des adultes pour le second ; ouverture sur l'espace social où l'éducation et la formation sont incluses parmi d'autres champs d'activité, pour le troisième).

53 Par exemple, Pujolle et al., 1985 ; Guihot, 1993.

${ }^{54}$ Par exemple, Germain, Gabriel, 1985 ; Compte, 1985c ; Van de Wiele, 1987. 
télé/visio-conférence ${ }^{56}$, numérisation de l'image ${ }^{57}$, multimédia ${ }^{58}$ ) et selon les types de pratiques $\left(\mathrm{EIAO}^{59}, \mathrm{FAD}^{60}\right.$, formation multimédias $\left.{ }^{61}\right)$. D'autres travaux rendent compte d'expériences pédagogiques qui ont été réalisées dans le domaine de la formation à distance grâce aux réseaux câblés de télévision ${ }^{62}$ ou font le point sur les questions posées par L'EAO ${ }^{63}$, notamment les questions didactiques et pédagogiques. La place plus importante que prend le terme «formation» par rapport à celui d' « enseignement» dans la désignation des dispositifs pédagogiques est révélatrice de l'amorce d'un changement de perspective dans la conception ingénierique des systèmes.

1.2.4. Années 1990 :

Hypermédias, technologies de

l'information et de la

communication (TIC) pour

l'éducation (TICE) et

formation ouverte et à distance

(FOAD)
La production de résultats s'organise selon trois axes principaux, qui étaient déjà présents durant les périodes précédentes sous forme de débats et de prises de positions individuelles ou collectives. À partir des années 1990, ces orientations tendent à être nommées, désignées, voire conceptualisés et s'organisent davantage comme des courants. L'un d'eux se structure autour des questions techniques, l'autre autour des langages, le troisième autour des usages.

Les travaux où dominent les questions techniques prennent une bien plus grande importance qu'auparavant ${ }^{64}$, car ce domaine tend à s'organiser

55 Par exemple, en sciences de l'information et de la communication, Moeglin, 1988. En sciences de l'éducation, Glikman, Chrétien, 1991.

56 Par exemple, Perin, Gensollen, 1992.

${ }^{57}$ Par exemple, Deken, 1983, 1984 ; Holtz-Bonneau, 1986 ; Quéau, 1986.

58 Par exemple, Chaptal, 1993 ; Crinon, Gautellier, 1997 ; dans la recherche francophone en sciences de l'éducation, Depover, Giardina, Marton, 1996 ; Giardina, 1999.

${ }^{59}$ Par exemple, La Passardière, Baron, 1991 ; Baron, Baudé, La Passardière, 1993.

${ }^{60}$ Par exemple, Lehnisch, 1981 ; Glikman, Baron, 1991 ; Lochard, 1995. Dans la recherche francophone en sciences de l'éducation, Henri, Kaye, 1985. En Angleterre : Bates, 1984, 1990.

61 Par exemple, Caspar, 1991.

62 Par exemple, Bertrand, Corset, Marmoud, 1986.

63 Par exemple, Perriault, 1983 ; Quéré, 1991 ; Demaizière, Dubuisson, 1992.

${ }^{64}$ Dans les périodes précédentes, le degré de technicité des machines et des systèmes sociotechniques était tel que ceux-ci restaient accessibles à des amateurs éclairés qui, dans leur milieu, passaient pour des «mordus», des «fous» de la technique audiovisuelle ou informatique. Entre les spécialistes et les néophytes, ils jouaient un rôle 
Note de synthèse - Technologies et formation

progressivement de manière autonome par rapport aux deux autres (langages et usages). La complexification technique qui requiert des compétences spécialisées développe une dynamique qui tend à se boucler sur elle-même. L'informatique atteint un tel degré de complexité que seuls les informaticiens spécialisés peuvent s'y intéresser, travaillant des sujets que seuls leurs pairs comprennent. C'est ainsi que se dessinent des approches différentes qui en se spécialisant, s'autonomisent, et en s'autonomisant se détachent les unes des autres, anticipant un mouvement de restructuration des frontières disciplinaires et des objets qu'elles étudient ${ }^{65}$.

Les travaux qui se centrent davantage sur les questions liées aux langages tendent à se développer autour des courants liés à la double dimension information et communication. Ils prennent appui sur la sémiologie de l'image développée dans les périodes précédentes (notamment à partir des écrits de Barthes, Eco, Metz, Veron) et autour de la sémiotique issue des travaux dans le domaine de la littérature et de l'art (notamment à partir des écrits de Peirce et de Greimas).

Les travaux qui s'intéressent davantage aux questions des usages commencent à se structurer plus fortement durant cette période autour des travaux fondateurs, en France, de J. Perriault (1989). Cette perspective se

de «traducteurs », de « passeurs », de « médiateurs ». Exerçant, pour la plupart, dans le milieu de l'éducation, ils en travaillaient les implications, les conséquences et pouvaient en évaluer les limites. C'étaient des périodes de «militantisme» à double sens : développer l'utilisation des technologies dans les pratiques de formation; intégrer la réflexion pédagogique dans la conception et l'utilisation de ces technologies. À partir des années 1990, cette situation change assez radicalement. L'informatique se complexifie extraordinairement (langages, hypermédias, réseaux). Les amateurs éclairés sont obligés de compter sur l'aide de spécialistes et peu à peu ceux-ci prennent position dans ce champ de recherche en détenant des savoirs et des savoir-faire spécifiques. Si cette spécialisation est indispensable, elle comporte cependant un travers, celui de structurer progressivement un champ particulier qui crée des disjonctions dans ce qui devrait se constituer en une "culture » commune. La difficulté alors est de créer des zones de "traduction", voire de «médiation», mais une telle dynamique dépend de la volonté des acteurs actifs dans les champs qui se diversifient, selon qu'ils structurent chaque champ sur des logiques de pouvoir ou sur des logiques de partage.

${ }^{65}$ Les Journées scientifiques «Hypermédias et apprentissages » qui donnent lieu à des publications régulières sous le même titre, sont un exemple des dynamiques à l'œuvre durant cette période, même si elles restent encore un espace de médiation entre des chercheurs intéressés par des questions diverses, mais dont le trait commun est de travailler sur le lien entre les aspects techniques et les aspects cognitifs, liés à l'activité d'apprentissage. Voir également : Bruillard, La Passardière, 1994. 
produit à un moment où la linguistique a commencé à donner des résultats dans le domaine de la pragmatique, où la littérature aborde la question du lecteur et de la part active qu'il a dans la production de l'œuvre. Dans un paysage de recherche kaléidoscopique qui analyse davantage les caractéristiques des outils proposés par les concepteurs et l'offre de formation, J. Perriault apporte une perspective renversée qui consiste à analyser les situations depuis l'usage qui en est fait. Il montre que tout ne se passe pas selon les projections et les intentions de l'instance ${ }^{66}$ de conception. Ses travaux et ceux qui se déploient dans ce courant d'analyse ${ }^{67}$ exercent une influence notable sur la conception ingénierique des dispositifs pédagogiques. Dans une période où le microordinateur rend l'informatique accessible au plus grand nombre, l'intégration de notions telles que celle d" "ouverture $»^{68}$, de $"$ flexibilité $»^{69}$, de gestion de la « distance $»^{70}, \mathrm{~d}^{\text {? }}$ " individualisation $»^{71}$ renvoie à une plus grande adaptation du dispositif à l'apprenant perçu comme «usager». Les interrogations pédagogiques se reposent avec une plus grande acuité encore, réinterprétant les principes et l'organisation du système existant ${ }^{72}$.

Cette période est marquée par une production très importante à un moment où il n'est plus possible de parler de «nouvelles» technologies, tant les nouveautés techniques deviennent une routine scientifico-industrielle. Les

\footnotetext{
${ }^{66}$ Nous empruntons ce terme aux travaux de C. Compte (1998) qui l'a développé dans le domaine de la communication pour analyser le média télévisuel et se référer à «l'instance de réalisation ». Ce terme permet de désigner non pas une personne ou un statut, mais une fonction remplie par plusieurs personnes de statuts différents exerçant parfois dans des espaces distincts d'un établissement ou d'une institution. Dans le contexte particulier de notre contribution, «l'instance de conception» peut inclure différents partenaires : le commanditaire, le financeur, les divers ingénieurs, informaticiens, designeurs, graphistes, tout comme le développeur industriel et le distributeur pour les dispositifs médiatiques ; les textes de cadrages, responsables de formation, enseignants, formateurs, tuteurs pour les dispositifs de formation.

${ }^{67}$ Par exemple, Agnel, 1994 ; Glikman, 1999.

${ }^{68}$ Par exemple, d’Halluin, 1993 ; d'Halluin, Haeuw, 1995 ; Carré et al., 1999.

${ }^{69}$ Par exemple, en France, Kuperholc, Mor, 1991 ; Kuperholc et al., 1993. Aux ÉtatsUnis, Steeples et al., 1994.

70 Par exemple, Caspar, 1991 ; Jacquinot, 1993 ; Perriault, 1996 ; Bernard, 1999. En Angleterre, Keegan, 1993.

${ }^{71}$ Par exemple, Prévost, 1994 ; Carré, 1997 ; Jézégou, 1998.

72 Par exemple, en France, Mangenot, 1999 ; Boullier, 1997, 2001. Au Québec, Tardif, 1998 ; Karsenti, Larose, 2001. En Belgique, Lebrun, Vigano, 1995 ; Lebrun, 2002.
} 
productions de rapports ministériels ${ }^{73}$ témoignent de l'intérêt du monde politique pour ces questions. Le système social a intégré les technologies à un point tel qu'il n'est plus possible de les penser de manière séparée. Certains travaux réalisés dans diverses disciplines diffusent l'idée qu'il s'agit d'un seul et même système sociotechnique ${ }^{74}$. L'usager y est analysé comme un acteur, doué de conscience et d'intentionnalité, capable de réaliser des choix motivés dans les interactions qu'il met en œuvre avec son environnement. On peut observer, dans les positionnements de recherche, le passage d'un intérêt pour l'activité d'enseignement «assisté » à une focalisation pour la «facilitation» des apprentissages. La notion de « dispositif » connait alors un regain d'actualité, car elle offre un cadre intégrateur dynamique capable de rendre compte des nombreux aspects, des tensions et des fluctuations dans le temps qui caractérisent toute organisation méthodique d'humains, d'instruments, de procédures et de stratégies en vue de fins particulières ${ }^{75}$.

Il est intéressant de remarquer que, parallèlement, au plan académique, face à la complexification du champ, certaines disciplines amorcent une redéfinition de leurs frontières: les sciences de l'information et de la communication tendent à se structurer explicitement en « interdiscipline » (Bougnoux, 1993 ; Miège, 1993 ; Ollivier, 2000), tandis que les sciences de l'éducation tentent d'élucider ce qui fait la spécificité de leur épistémologie (Charlot, 1995 ; revue Sciences de l'éducation pour l'ère nouvelle, 1998 ; Develay, 2001 ; Filloux, 2001).

1.2.5. Années 2000 :

Technologies, espaces

numériques de travail et

apprentissages

collaboratifs/coopératifs,

campus numériques

institutionnels ${ }^{76}$ aux statu internationaux ${ }^{78}$.
Cette nouvelle vague débute par la production exponentielle d'une littérature grise mise à disposition d'un large public sur le web, à propos des moyens techniques les plus récents et de leur utilisation en formation. Ces textes et documents proviennent de sites personnels (enseignants qui proposent leurs cours, amateurs éclairés), de sites divers, de sites ministériels ${ }^{77}$, d'organismes

\footnotetext{
73 V. Glikman (2002) signale quatre rapports importants dans cette seule décennie : Duhamel (1990) ; Serres, Authier, Lévy, Perriault (1991) ; Quéré (1994) ; Barbarant (1997).

${ }^{74}$ Rabardel, 1985 ; Flichy, 1995a/b ; Latour, 1999, 2001.

75 Jacquinot-Delaunay, Monnoyer, 1999 ; Choplin, 2002.

76 Par exemple les sites d'Algora, GEMME, Le Préau, OTE, sites universitaires, SFRS, Thot, Eifel (voir fiches descriptives dans ce même numéro).
} 
De plus en plus nombreux sont les chercheurs qui communiquent leurs travaux sur le web. Les revues électroniques se développent dans des disciplines diverses et des espaces publics ou privés qui commencent à proposer des aides à la recherche documentaire par le biais de méta-moteurs, des recensements et collections.

Cette productivité extrême (en quantité et en hétérogénéité), mise à disposition (réalisation et diffusion) dans des délais incomparables avec la publication sur support papier, constitue un changement important dans les formes de documentation, les temporalités du travail intellectuel et la prise en compte de la littérature grise dans les travaux de recherche.

Parmi cette littérature, la plupart des productions ont une orientation praxéologique (descriptions des produits de l'innovation technique ${ }^{79}$, descriptions de réalisations de l'innovation pédagogique ${ }^{80}$, rapports officiels d'instances politiques ${ }^{81}$ ). D'autres ont une orientation plus politique (revues d'associations ou d'organismes publics ${ }^{82}$ ). D'autres productions ont une orientation plus scientifique proposant des enquêtes ${ }^{83}$, des articles dans des revues électroniques ${ }^{84}$. Celles-ci s'organisent selon deux logiques distinctes, celle des abonnements payants qui recouvre les pratiques traditionnelles, celle des «archives ouvertes» qui met gratuitement à disposition du grand public la production scientifique ${ }^{85}$.

\footnotetext{
77 Par exemple celui de l'éducation nationale, dont certaines parties sur l'enseignement supérieur sont impulsées par la Direction de la technologie (Educasup, Formasup).

${ }_{78}$ Par exemple, les rapports de L'OCDE ou de l'UNESCO.

79 Par exemple les nombreuses descriptions de plates-formes, notamment celles de l'ORAVEP, d'Algora ou du Préau.

${ }^{80}$ Par exemple, de nombreux travaux canadiens en langue française sont publiés sur le web pour rendre compte d'expériences pédagogiques, notamment ceux du Réseau des centres d'excellence en télé-apprentissage impulsé par le groupe TACT à l'université de Laval.

${ }^{81}$ Par exemple, CPU, 1999 ; Averous, Touzot, 2002.

82 Par exemple, la revue de l'Association internationale des universités (AIU), hébergée par l'UNESCO.

83 Par exemple, INSEE, IREDU, ITEM-Sup (fédération dissoute en 2003).

84 Par exemple, la revue ALSIC, consacrée au domaine des langues étrangères ou la revue du Centre interuniversitaire de recherche sur la science et la technologie (CIRST).

85 Dans le domaine des technologies de l'information et de la communication pour l'éducation et la formation, création du site TEMATICE en 2003 (http://tematice.fr), répertorié en 2004 par le site du CNRS (http://hal.ccsd.cnrs.fr) (voir fiche descriptive dans ce même numéro).
} 
Note de synthèse - Technologies et formation

La période est encore trop récente pour anticiper une structuration de la production scientifique dans ce domaine, mais il est possible de voir se dessiner des axes de travail qui tendent à autonomiser davantage encore les trois courants repérés ci-dessus. Certaines orientations continuent de s'organiser autour des technologies émergentes et des pratiques qu'elles suscitent (les termes « virtuel » et «numérique » viennent compléter les termes « campus $»^{86}$ et " environnement $\|^{87}$ ). Il est cependant intéressant de constater qu'entre les périodes précédentes et celle-ci, l'intérêt pour les pratiques de formation, notamment la formation à distance, se centre davantage sur les apprentissages avec l'analyse des modalités de "l'apprentissage collaboratif » $^{88}$ ou de «l'apprentissage coopératif » ${ }^{89}$, ou encore la place qu’y occupe le «tutorat » $»^{90}$.

Au-delà donc des productions pléthoriques et hétérogènes qui se sont succédé au fil de ce demi-siècle, il reste des résultats suffisamment puissants pour constituer les bases théoriques de structuration d'un champ de recherche, au carrefour de plusieurs disciplines et de plusieurs sections académiques. Jusque-là émietté par des travaux épars, des définitions d'objets d'une grande diversité, des positions institutionnelles ou individuelles qui priment sur les intérêts scientifiques collectifs, ce champ n'a pas encore trouvé les moyens d'exister véritablement.

86 Par exemple, en France, Derycke et al., 1997. En Belgique, Peraya, 1999, 2002. Au Québec, Paquin et al., 1996 ; Paquette et al., 1997.

87 Par exemple, en France, les nombreux travaux sur «les environnements informatiques pour l'apprentissage humain » (EIAH). En Suisse, Dillenbourg, 2000. Aux États-Unis, Harasim, 1990 ; Wilson, 1997, 1999.

88 Par exemple, au Québec, Lundgren-Cayrol, 1996 ; Henri, Lundgren-Cayrol, 2001. Aux États-Unis, Guzdial et al., 1995 ; Coleman, Furey, 1996.

89 Par exemple, en France, Derycke et al., 1997 ; d'Halluin, 2001. Aux États-Unis, Adams, Hamm, 1990 ; Kagan, 1992 ; Ehrlich, Cash, 1996. Aux Pays-Bas, Kanselaar, Erkens, 1996.

90 Par exemple, en France, Annoot, 1998, 2001, 2004 ; Desprès, Leroux, 2003 ; Duplàa, Galisson, Choplin, 2003. Au Québec, Hotte, 1995. En Belgique, de Lièvre, Depover, 1999. 


\section{L'évolution des contextes contem- porains de la formation} Disjoindre technologies et pratiques est un

travers courant. Ce raisonnement revient à faire comme si les objets techniques étaient des entités autonomes, indépendantes des êtres et des situations, extérieures à toute contingence, réduites à leur seul aspect utilitaire. Cette représentation a longtemps brouillé la compréhension des phénomènes en jeu et rend difficile l'appréhension de l'immense ampleur des changements en cours. Cette deuxième partie de la note de synthèse invite le lecteur à un pensum que nous espérons salutaire. Dans un premier temps, en donnant des pistes pour une réappropriation d'une culture de la technique qui - loin d'être indépendante et circonscrite à une sphère pratique - peut être lue comme anthropologiquement constitutive de l'activité humaine. Dans un deuxième temps, en essayant de comprendre comment s'est produit le glissement entre une pensée de la technique, une tentative de fondation d'une théorie spécifique et une pratique ordinaire de la technologie qui se passe de pensée. Enfin, en repérant quelques-unes des caractéristiques du monde que la technologie contemporaine porte en elle et qui nous sont imposées, de fait, comme étant constitutives du monde contemporain.

Nous avons choisi de faire ce détour, pour tenter de mettre en relief les phénomènes culturels et sociaux qui se déploient en amont du domaine de la formation, mais qui contribuent largement à le configurer. Toute tentative de compréhension des phénomènes liés à l'intégration des technologies dans la formation nous semble en effet vaine si elle ne se situe pas dans ce contexte plus large.

\subsection{Technique et/ou technologie : la terminologie comme trace d'une culture anthropologique et sociale}

Le propos n'est pas de faire ouvre de lexicologue, mais d'interroger la terminologie en usage de manière à clarifier le réseau sémantique qui s'ouvre à partir de tel ou tel choix de termes. Or, le réseau sémantique ouvre lui-même sur un réseau de représentations mentales qui est en relation avec un réseau d'actes potentiels. S'interroger sur les mots en usage, c'est donc en même temps s'interroger sur les objets auxquels ils se réfèrent et sur les processus qu'ils contribuent à mettre en jeu.

La recherche sur l'usage des technologies dans la formation des personnes utilise une terminologie fluctuante qui tend à suivre l'évolution technique des 
supports. Au-delà des choix liés aux acronymes (NTIC, NTE, NTF, TIC, TICE, etc.), un débat de fond demeure entre deux termes : l'un, « technique », à l'apparence plus francophone ; l'autre, "technologie », à la consonance plus anglo-saxonne. Si le débat n'était que linguistique et culturel, il serait aisé à trancher. S'il perdure, c'est qu'il sous-tend des questions et des positionnements complexes. Dans cette partie de notre contribution, il s'agit moins de prendre position dans le débat, que de repérer des éléments signifiants, issus de la littérature, susceptibles d'aider à comprendre la complexité de la problématique.

Sans doute la technique plonge-t-elle ses origines aux sources de l'espèce humaine, non seulement comme fabrication et création au sens grec de sa racine (à la fois teucho $\hat{o}^{91}$, tuk.tos ${ }^{92}$, technè ${ }^{93}$ ) mais également comme élément

${ }^{91}$ Chez Homère : fabriquer, produire, construire. Teuchos comme désignation de l'outil est l'instrument par excellence dans le récit homérique : les armes. Pour C. Castoriadis $(1975,1978)$, Homère amorce le passage de ce sens à celui de causer, faire, être, amener à l'existence, détaché de la fabrication matérielle, mais pas de celle de l'acte approprié et efficace.

92 Dérivé de teuchô, tuktos signifie : bien construit, bien fabriqué et finalement achevé, fini, complété. Teketôn (le charpentier) est chez Homère l'ouvrier, l'artisan, le maittre dans une occupation, puis le bon constructeur, producteur ou auteur (source : C. Castoriadis, 1975, p. 803). Ce terme introduit donc une valeur positive et axiologique au faire.

93 Production, fabrication matérielle, mais surtout faire efficace, adéquat, qui perd le lien direct avec un produit. Il y a mise en valeur d'une manière de faire, de la faculté qui la permet, du savoir-faire productif relatif à une occupation. Quasi-synonyme d'épistémè chez Platon en tant que savoir rigoureux et fondé. Son sens est également connoté par une série d'oppositions à l'époque classique :

- technè-paideia, occupation lucrative opposée à l'apprendre désintéressé ;

- technè-tuchè, faire efficace et conscient opposé à un effet du hasard ;

- technè-physis, actualisation non naturelle d'un possible présent dans la nature opposé au réel brut, à ce qui, dans la nature, résiste au savoir-faire humain ;

Deux séries d'oppositions connotent également ce terme, qui peuvent cependant être analysées davantage en terme de tensions :

- technè-poiesis, le fabriquer matériel opposé à l'action de créer ; analysable comme une tension, puisque chez Homère le verbe poieô est presque synonyme de teuchô, dans la mesure où la création ne naissant pas ex nibilo, elle ne peut venir que de la physis (il y a un déjà là potentiel dans la nature) et/ou de la technè (savoir-faire qui permet d'actualiser ce potentiel par assemblage, ajustement, transformation).

- technè-praxis, le fabriquer matériel opposé au faire dans l'action ; analysable comme tension, puisque ces deux termes signifient une transformation, ce qui dans la physis pourrait être autrement, leur champ sémantique commun est celui du possible et de l'actualisation; en revanche, leurs fins diffèrent puisque 
constitutif du monde humain (Simondon, 1958), au même titre, par exemple, que le langage.

Les travaux d'anthropologues tels que A. Leroi-Gourhan (1943, 1945), B. Gille (1978), C. Parain (1979), A.-G. Haudricourt (1987) mettent en valeur l'interrelation permanente qui relie l'être humain et son milieu. Un milieu qui résiste mais en partie seulement, puisque l'activité humaine, dans son caractère technique corrélé au langage ${ }^{94}$ (ibid., 1964, 1965), altère, assemble, contourne, détourne, explore pour inventer à partir de l'existant, révélant de ce fait des "propriétés cachées de la nature qui auraient été condamnées à le rester à jamais» (Castoriadis, 1975, p. 804). Elle révèle surtout ce qui distingue les nécessités humaines en tant qu'espèce biologique de ses besoins en tant qu'être historique (ibid.). Pour C. Castoriadis, la technique qui a produit le phénomène d'industrialisation se dessine ainsi comme «le texte interminablement continué de l'impossible traduction du désir en visée réalisable » (ibid.).

Pourtant, selon L. Mumford (1934, 1950 ; 1967), la technique n'est pas à rattacher nécessairement à un objet. Elle est aussi rationalisation du travail humain et des rapports sociaux, organisation, division du travail et coopération rationalisée. Pour cet auteur, la technique n'est pas toujours matérielle, elle est également une pratique sociale réglée qui régule les rapports sociaux. Pour sa part, C. Castoriadis (1975) renforce cette perspective en pointant deux travers concernant la perception de la technique dans le système social : d'une part, tout ce qui en relève (faits et objets) est distingué des autres réalités sociales ${ }^{95}$; d'autre part, il y a identification entre le fait technique et son produit. Dans le même sens, A. Leroi-Gourhan $(1943,1945)$ met en valeur l'inscription de l'objet dans un "ensemble technique", un usage lié à l'histoire d'une communauté, à son niveau de technicité, à une connaissance, tout comme à une expérience à la fois collective et individuelle. Il est également le résultat d'une genèse qui, pour C. Castoriadis (1975) «met (...) à contribution la totalité de

si la technè vise l'ergon (le résultat, l'œuvre), la praxis, elle, s’inscrit dans le processus permanent de l'action humaine qui transforme le monde et par laquelle l'humain se constitue en tant que tel.

Pour les stoïciens, la technique est une hexis hodopoiétikè, un habitus créateur de chemin ; pour Aristote une hexis poiétikè, un habitus, une disposition permanente acquise, créatrice, accompagnée de raison vraie (source : C. Castoriadis, 1975, p. 804).

94 A. Leroi-Gourhan a mis en valeur la corrélation entre la capacité technique de l'être humain et son expression par le langage qui apporte à cette activité normes opératoires et statut social, tout en le situant dans un système de représentations et de valeurs à l'intérieur d'une communauté donnée.

${ }^{95} \mathrm{~J}$.-J. Salomon, par exemple, fustige cette tendance dans plusieurs de ses ouvrages. 
l'existence sociale de la collectivité qui le fait naitre » (ibid., p. 806). C'est en ce sens que l'ensemble technique, lié à l'ensemble économique et social d'une communauté, renvoie à sa vision du monde.

Le terme «technique » intègre donc cette constellation sémantique tissée au fil de l'histoire humaine et dont l'intrication avec l'ensemble des autres activités sociales est suffisamment complexe pour n'en être séparée - comme dans le cas du langage - que de manière artificielle.

Une première acception moderne du terme «technologie » remonte, pour J. Guillerme (1975), au XVIIe siècle, dans une période historique où la technique qui se transmet dans les ateliers, par imitation et répétition, prend un nouvel essor. Le développement des connaissances, en mathématique notamment, est lié à une plus grande rationalisation de l'activité humaine. Cette entrée des techniques dans la science moderne avec les arts mécaniques conduit à la naissance d'une nouvelle science, la technologie ${ }^{96}$, comme "description of crafts, arts or work-manship» (Blount $\left.{ }^{97}, 1670\right)$ ou encore comme « scientia artium et operum artis» (Wolff98, 1728). C'est durant cette période que J. Guillerme (1975) situe le passage d'une activité technique essentiellement guidée par l'empirisme à une activité qui devient objet de réflexion, de description, de codification et d'analyse ${ }^{99}$. Le rapport praticien-savant en est profondément - et peut-être irréversiblement - modifié. Comme le repère l'auteur, à partir du XVIIe siècle, le savant ne se contente plus de décrire les techniques passées, il lui arrive de les précéder et même de les engendrer.

Cependant, le développement de cette nouvelle science ne suit pas une trajectoire linéaire au fil des siècles. La tension entre l'instrumentalisation des savoirs qu'elle produit et le mépris de ces savoirs par les doctes, au prétexte de leur instrumentalisation, semble marquer l'histoire de la technologie comme « science des techniques» depuis son origine. J. Guillerme (1975) explique ainsi

96 Il est difficile de ne pas penser aux grands ingénieurs de l'Antiquité, à ceux de la période gréco-romaine, à des inventeurs tels que Archimède ou Léonard de Vinci qui a su allier, comme rarement dans l'histoire humaine, la technique, l'art et la connaissance scientifique. Cependant comme le remarque J.-J. Salomon (1992), jusqu'au Moyen âge, les ingénieurs étaient des "praticiens de talent» (ibid., p. 83). Lorsqu’à partir de la Renaissance, la formation technique s'allie à une éducation scientifique, la première est fortement liée à l'expérience individuelle et la seconde au hasard des rencontres dans la mesure où l'enseignement n'est pas institutionnalisé (ibid.).

${ }^{97}$ T. Blount, 1670, Glossographia (cité dans Guillerme, 1975, p. 821).

${ }^{98}$ C. Wolff, 1728, Philosophia rationalis sive logica (cité dans Guillerme, 1975, p. 821).

${ }^{99} \mathrm{~J}$. Guillerme cite d'autres exemples qui rendent compte de l'intérêt d'intellectuels du XVIIe siècle pour le développement de cette nouvelle science : Bacon (1627), Bosse (1643), Descartes (1648), Petty (1648). 
le sort contingent de cette discipline naissante : les initiatives les plus cohérentes dans le développement d'une «science des techniques » ont été assimilées par le génie militaire ; les autres ont connu « un sort incertain et point toujours prisé au sein d'académies scientifiques » (ibid., p. 821). Durant le XVIIIe siècle, des sociétés ${ }^{100}$ de techniciens prennent le relais du développement de cette nouvelle science et au début du XIXe siècle s'institutionnalise la formation des ingénieurs civils. La tendance vers l'instrumentalisation des savoirs et la professionnalisation des spécialistes dans ce domaine n'est donc pas un phénomène contemporain. En l'espace de deux siècles, le technologue s'est fait ingénieur. Au XIXe siècle, la systématisation des théories anglo-saxonnes ${ }^{101}$ s'inscrit dans un contexte social de développement de l'économie capitaliste et l'emporte contre une théorie allemande d'inspiration berckmanienne ${ }^{102}$ davantage tournée, pour J. Guillerme, vers l'univers artisanal dans le cadre d'un dirigisme économique. La technologie ne sera jamais aux techniques ce que la linguistique est aux langues ou la sémiotique aux langages.

Comme le note J.-J. Salomon (1992), à la plus ancienne acception du terme «technologie » comme logos de la/des technique(s) vient donc se substituer au cours du XIXe, puis du XXe siècle, une signification influencée à la fois par l'histoire de l'industrialisation et par l'usage prédominant du mot dans son acception anglaise. Ses racines étymologiques grecques, liées à la notion de «technique », restent indéniablement présentes dans le terme contemporain de «technologie », mais ce dernier rend davantage compte d'un phénomène socioéconomique sans précédent, celui de l'alliance irréversible de la technique, de la

100 J.-J. Salomon (1992) note que les ingénieurs civils étaient pour la plupart autodidactes dans le domaine technique et que le début de la révolution industrielle a été réalisé avec des chefs d'industrie et des cadres techniques qui n'avaient reçu aucune formation professionnelle instituée dans ce domaine. L'organisation d'associations professionnelles structure ce milieu et c'est à partir de cette période que commencent à s'institutionnaliser des formations spécialisées.

101 Par exemple, les traités d'économie manufacturière : C. Babbage (1832), A. Ure (1835) et E. Baines (1835) (cités par J. Guillerme, 1975, p. 821).

102 Johann Berckman délivra, à partir de 1772, les premiers enseignements qualifiés de «technologiques ». Cette formation dispensée à Göttingen était destinée à « éclairer des administrateurs appelés à faire des choix économiques »(Guillerme, 1975, p. 821). En 1777, il publie une Introduction à la technologie (Anleitung zur Technologie) où il argumente en faveur d'une rupture avec une conception liée à l'histoire des arts et des techniques. Son propos est de permettre à des professionnels de comprendre les principes des opérations qu'ils sont censés maîtriser, ainsi que les conséquences qui en résultent au plan économique. Il renforce donc cette rupture en reliant les techniques dans leur rapport pragmatique à la société (Salomon, 1992, pp. 73-74). 
science et de l'industrie dans le contexte d'une économie capitaliste dont le mot d'ordre est l'innovation.

\subsection{La technologie moderne : fruit d'une intrication croissante entre industrie et politique} spatiale et temporelle dans l'impact des objets techniques créés, des idéologies qui les sous-tendent et des conduites qu'ils suscitent.

\subsubsection{Une intrication scientifique, technique et industrielle}

Ce qui spécifie donc les technologies contemporaines par rapport à d'autres techniques utilisées par le passé, c'est à la fois : l'intrication technique, scientifique et industrielle dont elles sont issues ; le bouleversement social qu'elles contribuent à engendrer; le changement d'échelle D’après J.-J. Salomon (1992), les analyses de K. Marx et celles de J. Schumpeter, quoique différentes, restent complémentaires sur certains points et mettent à jour les processus selon lesquels l'innovation technique se fait moteur du changement dans les sociétés industrielles, constituant à la fois leur dynamisme et leurs dysfonctionnements.

Entre l'atelier de l'artisan, la manufacture et l'industrie moderne, ce qui a fait basculer le rapport humain au travail, c'est, pour ces auteurs, le moment où l'outil devient indépendant de la main qui le guide, rendant le machinisme impersonnel. C'est à partir de ce moment que les processus deviennent "séquençables», analysables, débouchant sur des applications routinisables, selon des lois étudiées d'après des procédés scientifiques. Devenue indépendante des limites humaines en terme de force musculaire et de fatigue psychique, la machine repousse à l'infini les limites de la production. Dans le développement de cette logique qui s'est avérée implacable au fil des deux derniers siècles, des liens de dépendance réciproque se sont noués entre invention technique, avancées scientifiques et production de capitaux.

\subsubsection{Valeurs matérielles et/ou valeurs éthiques?}

Cette intrication repérée par J. Habermas (1968, 1973) tend, selon ce philosophe, à dépouiller de leur dimension politique les choix sociaux qui sont réalisés au nom de la technique et au nom de la science. Pourtant, ce phénomène n'est pas nouveau. Comme le remarque C. Castoriadis $(1975,1978)$, pour Aristote, la technè séparée de la vertu éthique, conduit à concevoir la technique «comme instrument ancillaire et neutre» (ibid., p. 804). De même aujourd'hui, la science, la technique et l'industrie sont trois domaines de l'activité humaine qui apparaissent tout aussi indifférents au problème des valeurs éthiques (Salomon, 1992). Pourtant, malgré la dénonciation véhémente 
de chercheurs tels que J. Ellul (1954) contre l'illusion de la neutralité et de la pure instrumentalité de la technique, nombre de secteurs de la recherche continuent à œuvrer en séparant, de manière inconsciente ou revendiquée, recherche et réflexion éthique.

L'efficacité étant l'aune à laquelle est mesurée une technique, celle-ci en constitue la valeur intrinsèque. Une technique est «bonne» si elle est efficace. Reste à savoir : efficace par rapport à quoi ? Dans quel but? Et par rapport à quelle partie de la population à l'échelle locale ou planétaire ? Le masquage - ou l'oubli permanent - des interrogations portant sur les finalités et les conditions sociales du développement technologique dans les sociétés post-industrielles signale une difficulté constitutive et non pas accidentelle. Il n'est pas aisé, en effet, de passer d'une analyse fonctionnelle réalisée à une échelle très localisée (le choix d'une technique par rapport à une autre dans un contexte donné) à une analyse compréhensive prenant en compte le contexte même de l'instrumentation, ses acteurs et son évolution dans le temps. La première tend à privilégier l'efficacité immédiate sous couvert de neutralité, la seconde réintroduit dans le projet individuel la responsabilité éthique envers le collectif et la perspective d'un projet politique. Étendue à l'échelle sociale, voire historique, l'analyse compréhensive des conséquences, des significations et des valeurs finales de l'instrumentation pour les diverses activités humaines est difficile à mener et complique les décisions. L'attention aux acteurs sociaux et aux situations réelles d'usage arrive ainsi à ébranler le fondement des logiques purement rationnelles qui sous-tendent une instrumentalisation de l'action en prétendant s'en tenir à des descriptions objectives des buts, des tâches et des procédures.

La «Question de la Technique » telle qu'elle a été posée par M. Heidegger $(1954,1958)$ reste une référence incontournable. De même, son analyse de l'ambivalence essentielle de l'activité technique en tant que mode particulier d'expression humaine. Pour ce philosophe, la caractéristique essentielle de la technique n'est «absolument rien de technique» (ibid., p. 9), elle ne se réduit pas à un moyen méthodique conçu en vue d'une fin. Elle est un mode humain de rapport au monde, une forme particulière de "dévoilement» de notre manière «d'être-là ». Mais ce dévoilement est ambivalent : il oscille entre la «pro-duction» de la nature qui révèle et rassemble ses propriétés dans le faire traditionnel et son «arraisonnement» brutal par l'exploitation industrielle et le calcul rationnel. Quand ils imposent l'instrumentalité comme seule fin, ces deux modes sont dangereux car ils nous enferment dans la seule volonté de maittrise et bloquent toute possibilité d'interrogation sur nous-mêmes et notre "existence ». Mais rien ne nous empêche de les mettre en question ou de nous 
laisser enfermer "dans une morne contrainte qui nous [obligerait] à nous jeter tête baissée dans la technique ou, ce qui reviendrait au même, à nous révolter inutilement contre elle et à la condamner comme une œuvre diabolique » (ibid., p. 34).

Cette vision de la technique, non comme simple moyen mais comme forme ambivalente de dévoilement de notre rapport au monde, a été reprise dans des perspectives plus politiques, notamment par J. Habermas (1968), J. Baudrillard (1968), C. Castoriadis $(1975,1978)$. Elle permet de saisir comment, livrée à sa seule logique, la technique devient un sous-système social qui tend à créer son propre monde.

C'est ainsi que la technologie contemporaine, en rationalisant les conditions d'existence et le rapport au monde des sociétés post-industrielles, s'érige en idéologie et masque, sous couvert de progrès matériel, «l'institutionnalisation d'une domination qui n'est plus reconnue comme domination politique » (Habermas reprenant les arguments d'H. Marcuse, 1968, p. 5).

On peut alors se demander si l'expansion technologique planétaire actuelle correspond à un processus anthropologique de "complexification-conscience » et de «convergence » mentale qui double l'évolution de la biosphère d'une « noosphère » réflexive unificatrice (Teilhard de Chardin, 1955, 1959) ? Ou bien si elle correspond davantage à un processus mécanique d'autonomisation irréversible (Ellul, 1954, 1988) qui replonge - au lieu de la soustraire - l'essence de la technique dans la seule ontologie du faire (Castoriadis, 1975, 1978). Dans les deux cas, la coexistence des sciences et des humanités est à méditer sur deux points : « le savoir d'agir sur la nature et le savoir de nous penser nous-mêmes » (Salomon, 1992, p. 181) ; le rapprochement de deux formes de rationalité, traditionnellement présentées comme antinomiques, «la poursuite du savoir et la quête du sens » (ibid., p. 184).

\subsubsection{Un bouleversement social sans précédent}

Le rythme de l'innovation technique contemporaine imprime une cadence de changements quasi-continue dans la diversité des espaces professionnels et privés des personnes. Au cours de l'histoire humaine, l'adaptation à l'innovation technique s'est faite sur des bases très différentes de celles d'aujourd'hui. D'abord, les temporalités d'adaptation étaient bien plus longues que celles auxquelles sont confrontés les individus depuis environ un siècle ou même un demi-siècle ${ }^{103}$. D'autre part, jusqu'à la période industrielle

${ }^{103}$ Dans la transmission des signaux : plusieurs milliers d'années ont été nécessaires pour passer du papyrus (fragile et nécessitant les deux mains) au parchemin (plus solide, pouvant être stocké et libérant une main); plus de douze siècles pour passer du 
(fin XVIIIe, début XIXe), les personnes touchées par les inventions techniques étaient restreintes à un tout petit cercle d'individus (savants, intellectuels, artisans, puis professionnels d'un secteur de l'industrie). Aujourd'hui, le temps de passage entre l'invention technique et l'innovation comme phénomène d'appropriation sociale s'est considérablement raccourci et touche un éventail de personnes bien plus large. Il y a donc là un mouvement contraire aux sociétés antérieures, dans la mesure où les changements biologiques, sociaux et culturels impulsés par les technologies sont intrinsèques au système social. Comme le remarque J.-J. Salomon (1992), l'état d'équilibre et de stabilité des sociétés traditionnelles n'était rompu que par des phénomènes extérieurs (guerres, invasions, catastrophes naturelles, épidémies). L'une des caractéristiques de la société industrielle est d'ajouter à ces causes externes, les mutations internes qui rendent permanente l'instabilité des environnements professionnels et privés et placent au rang des « routines » (ibid., p. 92 et 99) les mutations techniques, sociales et culturelles.

Le changement ainsi inscrit dans la structure économique, sociale et culturelle des sociétés industrialisées tend à rendre de plus en plus difficile l'isolement individuel et collectif. Rares sont les personnes et les nations qui ne s'inscrivent pas aujourd'hui - de gré ou de force - dans le mouvement international de développement des technologies. Cette impossibilité d'isolement et cette difficulté à résister à ce mouvement ne vont pas sans un coût social dont on ne mesure sans doute pas encore complètement les conséquences.

\subsubsection{Un changement d'e- chelle spatiale et temporelle des impacts sociaux du "progrès 》 \\ $\mathrm{Si}$ l'alliance entre science et industrie comporte, pour nombre de personnes, une dynamique positive d'évolution et de progrès, elle génère parallèlement une misère et une souffrance qu'il est de plus en plus difficile de ne pas prendre en compte ${ }^{104}$.}

parchemin au papier (qui permet à l'imprimerie de se développer) ; cinq siècles pour passer des messages écrits au télégraphe; près de 70 ans pour adopter le téléphone. Les générations contemporaines se seront adaptées en moins de 50 ans à plus d'innovations que toutes les générations passées (Source : Kittler, 2002).

104 J. Baudrillard (1968) dénonce la «fausse conscience du progrès » que génèrent «les perfectionnements mineurs, complication et systèmes annexes » qui «masquent l'urgence des transformations essentielles » (ibid., p. 176). Plus loin, il ajoute : «il est difficile d'évaluer le déficit global que constitue pour l'ensemble d'une société cette diversion aux conflits et aux besoins réels sans la technique, elle-même asservie à la mode et à la consommation forcée. Ce déficit est colossal» (ibid., p. 178). Il conclut 
Note de synthèse - Technologies et formation

La dépendance entre l'être humain et la machine est aujourd'hui telle que l'organisation sociale du travail tend à exiger du premier les capacités de la seconde, en termes de durée des temps de travail et de cadence, de perfectionnement continu et indéfini et au nom d'une conception de l'efficacité dont l'aune est le rendement productif. Il s'agit de «consommer le temps à des fins exclusivement utiles » (Salomon, 1992, p. 203), ce qui réduit la place des "plages de temps libre conquises sur l'usage fonctionnel du temps » (ibid.).

Comme le montre C. Dejours (1998) par ses enquêtes, les coûts sociaux non mesurables par les indicateurs économiques et statistiques n'en sont pas moins existants. Le travail aliéné perdure, malgré les développements technologiques. Il serait trop facile de dire «à cause » du développement technologique, car la caractéristique du travail aliéné, c'est précisément son absence de signification pour la personne qui le produit et non pas l'outil avec lequel elle le produit ou l'environnement dans lequel elle le produit.

Cet état de fait conduit à s'interroger sur la valeur de ce changement technique et la manière dont il est finement relié aux changements sociaux et culturels. Ce ne sont pas seulement les caractéristiques techniques d'une société qui changent, indépendamment des structures sociales et culturelles, c'est aussi l'ensemble du système social et culturel qui s'actualise avec ses langages et ses codes, ses modalités d'échanges et de développement, ses productions artistiques et matérielles. La question spécifique que posent les technologies aujourd'hui c'est, par le biais entre autres du savoir et de la connaissance, le nouveau rapport au pouvoir qu'elles instituent en modifiant les structures mêmes de pouvoir, dans la mesure où elles installent une relation de dépendance systématique entre science, industrie et pouvoir. Dans le lien moderne entre ces trois pôles de l'activité sociale, l'innovation n'est plus seulement un moyen d'atteindre les objectifs qui pourraient être fixés dans le cadre d'une politique sociale par exemple, elle est devenue une fin en soi, un système institué en vue de réaliser toujours un plus grand profit, indépendamment des coûts sociaux.

Le domaine de la formation n'échappe pas à cette dynamique sociale. Le temps de l'apprentissage est un temps qui se doit d'être, lui aussi, productif. Dans cette conception utilitaire, apprendre revient à modifier des conduites, en termes de savoirs et de savoir-faire dans une temporalité rentable, c'est-à-dire courte. Face à l'obsolescence rapide de certains savoirs, la personne est mise en

ainsi un chapitre : "Tout se meut, tout change à vue, tout se transforme, et pourtant rien ne change. Une telle société, lancée dans le progrès technologique, accomplit toutes les révolutions sur elle-même. Sa productivité accrue ne débouche sur aucun changement structurel » (ibid., p. 217). 
situation d'apprentissage permanent sur le modèle de la consommation de biens matériels. Comme l'argumente G. Lerbet (1992, pp. 15-16), le temps de la schola - celui de la rationalité hétéronome, de "l'école du dehors» (ibid.) - prend le pas sur le temps de la scholè - celui de l'oisiveté qui permet de mûrir les processus, de «féconder l'esprit pour ensemencer des compétences » (ibid.) et qui offre un espace à «l'école du dedans » (ibid.).

C'est ainsi que le savoir et la compétence peuvent être transmués en biens de consommation, l'éducation et la formation en modalités d'investissement et sources de crédits financiers. Une telle conception conduit à fabriquer des formes d'enseignement rentables, aisément et rapidement transportables sur les réseaux câblés d'une planète qui devient un formidable réservoir de clientèle. Ce n'est donc pas tant la technique qui pose problème que le projet social et politique au service duquel elle est placée. C'est pourquoi ces différents éléments de la culture et de la société nous paraissent devoir être étudiés ensemble et non séparément.

Cette condition paraît d'autant plus importante que le passage d'une échelle locale à une échelle planétaire (Lévy, 1987, 1990) permet une diffusion large et rapide (d'idées autant que de virus) et donne à chaque acte et à chaque engagement un caractère de responsabilité plus important que jamais dans l'histoire humaine. Le pouvoir des êtres humains sur les limites de leur propre condition en ce qui concerne par exemple leur reproduction, leur mobilité, leur capacité de production et de destruction, mais aussi sur leur milieu de vie et leur environnement naturel et social, n'a plus rien de comparable avec les périodes historiques antérieures. La capacité d'éducation et de formation de toutes les générations n'échappe pas à cette modification qui atteint peu à peu tous les secteurs de la vie. L'ordre de grandeur des conséquences des décisions sociales et politiques est inédit. Dans une contribution récente et à partir des lectures de Heidegger (1954), Habermas (1968) et Jonas (1979), M. Linard (2003) pose tout un ensemble de questions qui invitent le chercheur à repenser ses modes mêmes d'interrogation et d'investigation, ainsi que son rapport au monde moderne.

2.3. Enjeux sociaux, économiques et politiques liés aux technologies contemporaines dans le domaine de la formation des personnes
Malgré tous les désordres que les changements économiques et sociaux évoqués ci-dessus révèlent, il peut être fructueux d'analyser la situation contemporaine, non pas en termes manichéens et moraux qui pousseraient à rejeter les technologies et leurs dynamiques sociales, mais en réactualisant la distinction marxienne entre 
Note de synthèse - Technologies et formation

«moyen matériel de production» et «mode social d'exploitation». Ce ne sont pas les ordinateurs qu'il faut brûler, comme le feraient des luddistes, mais les modes de travail et d'échanges qui sont à explorer et à réfléchir sur de nouvelles bases.

Dans le domaine de la formation des personnes la question est loin d'être une évidence tant les niveaux de production de ressources et d'offre de services d'une part, et d'autre part, les niveaux d'intrication avec les autres dimensions sociales sont complexes (politiques et économiques notamment). L'analyse des phénomènes d'industrialisation de l'offre de formation met en valeur les liens de dépendance réciproque qui tendent à s'instaurer dans ce domaine comme dans d'autres et pose la question de la responsabilité des chercheurs et des praticiens.

\subsubsection{L'industrialisation de l'offre de formation}

Comment croire encore aujourd'hui qu'aux

côtés de l'armement, des télécommunications, de l'alimentation ou encore de la santé, l'éducation et la formation échappent aux dynamiques engendrées par les technologies dans ces domaines de l'activité humaine?

Analyser les phénomènes sociaux qui conduisent à faire de la formation une marchandise et conceptualiser cette approche (Moeglin, 1988 ; Miège, 1989) s’inscrit dans la lignée des travaux qui s'interrogent sur les phénomènes d'industrialisation culturelle ${ }^{105}$ et d'industrie médiatique ${ }^{106}$, depuis les années 1970. Ce courant de recherche met en valeur un certain nombre de phénomènes sociaux qui conduisent à réinterroger les rapports que le système éducatif entretient avec le système social, à partir d'un jeu de tensions entre sphère industrielle et sphère éducative, au plan des logiques, des types de discours, des finalités, des modèles de société (Fichez, 1993 ; Moeglin, 1998). Ces analyses permettent de dépasser des représentations du monde industriel provenant en partie des analyses marxiennes des étapes de la production industrielle ${ }^{107}$, dans la mesure où les technologies numériques modifient en

105 Par exemple, Horkeimer, Adorno, 1974 ; Huet et al., 1978 ; Lacroix et al., 1979 ; Tremblay, 1990.

106 Par exemple, Flichy, 1980 ; Mattelart, Piemme, 1980.

107 Celui-ci analyse le passage (applicable au monde de la formation) entre la situation artisanale où chaque artisan produit lui-même, en s'appuyant sur un certain nombre d'outils, la totalité d'un objet. La manufacture où un plus grand nombre de personnes interviennent selon une division du travail qui permet de prendre en charge, au gré des disponibilités, préférences et compétences de chacun, une partie des opérations dans 
profondeur le paysage économique et social. Elles font entrer le monde de la formation dans un vaste système de relations sociotechniques en réseau qui implique des modes d'intervention différenciés au niveau de la production, l'approvisionnement, l'entretien, la maintenance, etc. Or, plus ce système est complexe, plus il est vulnérable (pannes, bugs, etc.), nécessitant un investissement humain important sur des postes techniques négligés jusque-là (informaticiens de réseau, webmasters, programmeurs, techniciens, etc.). En prolongeant la pensée de L. Mumford (1934), il est possible d'affirmer que les technologies ne sont pas seulement des objets, des machines et des biens matériels, elles sont aussi des biens immatériels, des langages, des programmes, des techniques de gestion, d'organisation et de décision, qui reconfigurent l'espace culturel et social. La réflexion sur les questions liées aux processus d'industrialisation de la formation permet de comprendre des phénomènes devenus extrêmement complexes. P. Moeglin (1998) épingle ainsi l'oubli de la pédagogie dans nombre des développements techniques des moyens. Il impute cet oubli à un processus de «contestation de la valeur d'usage de la formation, dans le contexte de délégitimation des grandes finalités éducatives, traditionnellement liées aux principes de la connaissance recherchée pour ellemême ou mise au service du progrès culturel et social » (ibid., p. 246). Seul un travail rigoureux sur ce type de processus peut permettre de dévoiler les idéologies sous-jacentes à des choix de société qui, sous couvert de modernité, tendent à se faire avec l'aval des individus, parfois les plus engagés dans une logique de service public.

\subsubsection{Une relation de dépendance réciproque entre pouvoir politique et production scientifique}

Déjà analysé par J. Habermas $(1968,1973)$ qui fustigeait la «scientifisation de la politique» et la "politisation de la science ", qualifié par M. Callon (1998) d' «imbroglio scientifico-politique » (ibid., p. 262) et d' " enchevêtrement» (ibid., p. 264), ce lien de dépendance, généré en partie par le développement des technologies, est sans doute ce qui distingue le plus la société contemporaine.

La production scientifique portant sur les technologies ne peut plus se faire sans des moyens matériels considérables. Parallèlement, les décisions politiques liées à l'intégration de technologies coûteuses, et dont l'impact peut être déterminant pour l'avenir, ne peuvent plus se prendre selon une tactique essaierreur. D'une part, la recherche scientifique est liée aux soutiens financiers qui la font vivre ; d'autre part, les instances politiques sont de plus en plus liées aux

une succession qui garde la maitrise de l'ensemble. L'industrie où la production dépend de machines sur lesquelles l'humain n'intervient que pour réguler les performances. 
Note de synthèse - Technologies et formation

avis de scientifiques qui interviennent alors sur la base de leur expertise dans un domaine spécialisé.

Les enjeux sont donc extrêmement importants pour une société à visée démocratique, car cela pose la question des formes de la régulation : celle des capitaux destinés à la recherche ; celle de la validation et de la désignation de l'expertise ; celle, enfin des pouvoirs réciproques de la politique et de l'expertise. La régulation des capitaux destinés à la recherche concerne à la fois leur provenance, leur destination et les modalités de la sélection. La régulation de la validation et de la désignation de l'expertise concerne le fonctionnement des instances académiques et des conditions de son ouverture au monde entrepreneurial et politique. La régulation des pouvoirs réciproques de la politique et de l'expertise est liée à la reconnaissance des compétences, mais aussi des limites ${ }^{108}$ intrinsèques à ces compétences et à l'exercice de l'expertise ${ }^{109}$.

Dans le domaine de la formation des personnes, ce contexte entraine un certain nombre de questions sur le statut de la connaissance. Deux situations extrêmes sont observables : soit celle-ci est perçue seulement comme une valeur marchande et elle obéit aux règles d'un marché, en fonction des profits qu'elle est supposée apporter ; soit elle est également considérée comme ayant une valeur autre que marchande et il est possible d'alimenter des espaces sociaux non directement productifs au sens où l'entend la logique industrielle. Dans le premier cas, le monde de la formation est pleinement industrialisable et la connaissance peut être traitée comme un objet d'investissement, de capitalisation et de profit. Dans le second cas, il y a place pour d'autres formes de représentation du monde et d'autres modes d'échange que l'acquisition et la vente de ce bien, tout comme les services relatifs à ce bien.

À une époque où le monde politique commence à appréhender une représentation écologique du monde et une notion telle que le « développement

108 Un certain nombre de travaux en sociologie des sciences (Morin, 1982 ; Latour, 1994 ; Callon, 1998) ont révélé la " cécité » liée à chaque domaine de connaissance.

${ }^{109}$ J.-J. Salomon cite les travaux de C. Snow (1960) qui « a dénoncé l'euphorie qui aliène le jugement des scientifiques» (Salomon, 1992, p. 151), jugements «d'autant plus dangereux » qu'il revêtent «l'habit technique» qui «leur confère l'autorité d'un jugement objectif» (ibid.). J.-J. Salomon fustige deux travers des scientifiques et d'un système social qui survalorise leur position d'expert : le premier consiste en "un attachement si exclusif à (leur) propre point de vue qu'il(s) en perd(ent) toute objectivité » (ibid., p. 152) ; le second consiste à laisser croire que leur expertise dans un domaine très précis les qualifie « pour traiter de n'importe quel sujet » (ibid.). 
durable», il est possible d'envisager l'intelligence humaine comme une ressource naturelle à ménager plus qu'à exploiter.

Ces diverses interrogations, posées dans cette contribution de manière très contrastée, visent à délimiter le paysage social dans lequel s'inscrivent les interrogations sur les phénomènes suscités par l'intégration des technologies dans les dispositifs de formation. La question qui se pose au chercheur est de savoir comment il peut à la fois ne pas nier les contraintes économiques et les stratégies industrielles dans le domaine où il exerce, tout en restant vigilant à dévoiler les dérives qui portent atteinte aux intérêts individuels et collectifs spécifiques au domaine qui est le sien. Cette fonction de la recherche dans l'intelligibilité des processus liés à l'instrumentation des apprentissages peut contribuer à réguler les tendances technocratiques d'un système social et politique largement conduit par une économie de marché.

\subsubsection{Activité scientifique dans le domaine de la forma- tion et responsabilité sociale}

Une telle perspective d'analyse du contexte social et de la place qu'y occupent les technologies conduit à réinterroger la position wébérienne de neutralité du savant. Si l'objectivation reste une posture qui fonde l'activité scientifique, l'implication dans l'analyse des conséquences sociales de la recherche ne peut pas être négligée.

Nombre de travaux dans ce domaine dénoncent la croyance en la neutralité des technologies que ce soit dans son rapport à l'espace anthropologique et culturel $^{110}$, à l'espace social et politique ${ }^{111}$, à l'espace cognitif et plus généralement formatif ${ }^{112}$. Une autre série de travaux désacralisent le mythe de la neutralité de la science ${ }^{113}$. La critique de la rationalité occidentale (Jullien, 1996), et de la rationalisation généralisée des activités (Habermas, 1968), engage à une grande prudence. Dans cette perspective critique, J.-J. Salomon (1992) met en exergue l'une des faiblesses de l'organisation rationnelle qui «ne porte pas en elle-même sa destination ». Pour sa part, M. Linard (2003) analyse l'incapacité de contrôle des effets d'amplification et de résonance que produisent les technologies. Reprenant les thèses de H. Jonas (1979), elle met en exergue la domination de l'agir sur la capacité de prévoir et de juger, éthiquement, des conséquences de l'agir. C'est le risque que courent les dispositifs

110 Par exemple, Mumford, 1934, 1967 ; Leroi-Gourhan, 1943, 1964 ; Simondon, 1958.

111 Par exemple, Heidegger, 1954 ; Ellul, 1954 ; Marcuse, 1964 ; Habermas, 1968 ; Castoriadis, 1975 ; Salomon, 1992 ; Sfez, 2002.

112 Par exemple, Linard, 1973, 1989 ; Jacquinot, 1977, 1985 ; Compte, 1985a ; Moeglin, 1988, 1998.

113 Par exemple, Prigogine et Stengers, 1981; Jurdant, 1998 ; Latour, 1994, 2001. 
sociotechniques développés dans le cadre de la formation des personnes. En matérialisant, de manière systématique et continue le possible, «le sens et la valeur de ce qui est réalisé se noient dans la seule efficacité technique » (ibid., p. 168). C'est sans doute l'une des raisons pour lesquelles nombre de chercheurs intéressés pas les questions liées à l'instrumentation des apprentissages partagent le sentiment d'une réitération des problématiques exprimées au fil du temps sous la diversité d'une terminologie actualisée par les effets de mode. Les technologies évoluent et les mots pour les qualifier changent, mais les problèmes de fond, concernant la conduite réussie des apprentissages, demeurent.

La recherche sur les usages ${ }^{114}$, tout comme un ensemble de travaux empiriques irréprochables ${ }^{115}$, repose sérieusement la question du déterminisme technologique et de son caractère inéluctable. Ces travaux mettent en valeur l'activité d'appropriation et de détournement propre à l'usage naturel des outils en montrant que tous les possibles dont les projets techniques sont porteurs ne sont pas nécessairement actualisés. L'usager adapte en permanence les environnements sociotechniques à ses besoins, selon ses acquis et selon le type de conduite qui motive ses choix. Mettre l'accent sur les logiques de l'appropriation sociale permet de réintroduire, dans la logique technocratique de développement outrancier des technologies, l'interrogation sur les valeurs sousjacentes aux projets politiques et sociaux qui accompagnent le développement technologique d'une société.

\section{Incidences sur la recherche en sciences de l'éducation}

De nombreux secteurs scientifiques s'intéressent aujourd'hui aux phénomènes psychocognitifs, sociaux, économiques, ingénieriques et techniques, liés à l'intégration des technologies dans la formation des personnes. Que peuvent apporter les sciences de l'éducation dans ce vaste champ, tout en échappant à l'obsolescence des modes et des terminologies liées à l'évolution rapide des artefacts?

Quatre orientations nous semblent aujourd'hui essentielles : une exigence de cumulativité et un travail de mémoire ; une approche anthropocentrée effective dans les perspectives de recherche; une approche transversale et interdisciplinaire des interrogations et des problématisations ; une prise en compte des perspectives axiologiques et praxéologiques.

114 Par exemple, Perriault, 1989 ; Flichy, 1995 ; Stiegler, 1997.

115 Theureau, 1992 ; Clot, 1995 ; Rabardel, 1995 ; Leplat, 1997. 


\subsection{Une exigence de cumulativité et un travail de mémoire}

Le constat a été fait de manière répétée : dans le domaine de l'éducation et de la formation, l'innovation technique produit des discours qui se présentent le plus souvent comme inédits, la nouveauté d'un objet venant balayer la connaissance élaborée à partir de ceux qui l'ont précédé. Si les raisons de ces amnésies partielles sont tout à fait compréhensibles, voire analysables, un travail de mémoire, collectivement assumé, orienté par une exigence de cumulativité, permettrait d'enrayer ce phénomène récurrent.

Pour cela, trois types de discours sont à mettre en relation, tout en étant distingués : les textes politiques et institutionnels ; les discours éducatifs qui accompagnent le développement d'outils et de dispositifs ; les résultats théoriques répertoriés sur des questions précises.

L'analyse des textes politiques et institutionnels permet de réinscrire l'action de formation dans son contexte sociopolitique ${ }^{116}$. Parallèlement, l'analyse des discours des acteurs éducatifs ${ }^{117}$ qui accompagnent le développement d'outils et de dispositifs permet de situer l'action de formation dans un système idéologique au sens large du terme (intentions, enjeux et moyens projetés, finalités, catégories qui président à l'évaluation et à la régulation de l'action).

Ce double travail d'analyse permet de comprendre à quel moment et à quelles conditions des décisions politiques ont pu devancer, impulser, accompagner ou bloquer des dynamiques d'innovation en formation ${ }^{118}$. Systématisé et conservé, il permet d'élaborer une mémoire commune.

En recherche, le repérage problématisé de résultats produits en France et à l'étranger, dans et en dehors de la discipline, n'est pas assuré de manière systématique. Face à la publication exponentielle de résultats hétérogènes et éparpillés dans des publications difficilement accessibles, de telles synthèses offriraient pourtant la possibilité de confronter utilement les cadres théoriques et les approches méthodologiques. Elles permettraient de rendre visibles les convergences et divergences entre des disciplines qui travaillent le plus souvent de manière isolée. Alliée à la connaissance des terrains de la formation et de la culture pédagogique, cette connaissance interdisciplinaire conduirait à la formulation de questions qui ne sont traitées par aucune discipline prise isolément.

Cette attention temporelle, dans un souci de conservation de traces et de cumulativité de résultats, ouvrirait deux perspectives. Une perspective socio-

116 Par exemple, Dieuzeide, 1985 ; Glikman, 1989 ; Papadoudi, 2000.

117 Par exemple, Lemaire, Marquet, Baillé, 1997 ; Albero, 1998, 2000.

118 Par exemple, Thibault, 2003. 
historique permettrait de constituer une mémoire politique et pédagogique de référence. Une perspective épistémologique mettrait en valeur trois pistes de travail qui n'apparaissent pas de manière évidente dans la profusion synchronique : l'analyse du rapport entre "savoirs théoriques » et "savoirs d'action » (Argyris, 1970 ; Argyris, Schön, 1974 ; Schön, 1983 ; Barbier, 1996) ; l'analyse du rapport entre "sémantique de l'intelligibilité » et «sémantique de l'action» (Barbier, 2000) ; enfin, l'analyse critique des articulations entre cadres théoriques et choix méthodologiques.

\subsection{Une approche anthropocentrée dans les perspectives de recherche}

Sur la base d'une abondante revue de la littérature, P. Rabardel (1995) différencie approche «technocentrée » et approche « anthropocentrée » dans la recherche sur l'activité humaine instrumentée. En se centrant sur l'optimisation de l'objet technique, la première pense l'activité humaine comme «résiduelle», au service du fonctionnement de l'outil. La seconde analyse le fonctionnement de l'outil non comme « auxiliaire », mais constitutif de l'activité humaine.

Toute recherche qui focalise son attention sur les pratiques et les acteurs de la formation est, de ce fait, nécessairement anthropocentrée. Elle est amenée à prendre en compte la totalité sociotechnique d'environnements mixtes, non seulement numériques, mais aussi naturels et sociaux. Un tel choix épistémologique ne va pas de soi et de nombreuses questions théoriques et méthodologiques se posent (nous en évoquerons quelques-unes aux deux points suivants). Ce type de recherche est, par ailleurs, à positionner en complémentarité avec d'autres approches, celle des EIAH par exemple pour laquelle, «l'utilisateur (...) est sous le contrôle de la machine et non l'inverse » (Balacheff, 2001). Ce qui n'empêche pas de reconnaître comme "principal verrou » la nécessité de prendre en compte le double mouvement « du sujet qui évolue au cours de l'apprentissage et de l'environnement [numérique] qui doit évoluer pour accompagner cette évolution » (ibid.).

Le fait qu'une partie de la recherche en sciences de l'éducation ne s'intéresse aux technologies que de manière marginale, en se focalisant davantage sur les systèmes d'interactions à finalité de formation n'est pas un hasard. Les travaux portant sur les pratiques d'autoformation en contexte institutionnel ${ }^{119}$, les centres de ressources ${ }^{120}$, ou encore le vaste champ de la formation ouverte et à

119 Par exemple, Carré, 1991, 1992 ; Carré, Moisan, Poisson, 1997 ; Albero, 1998, 2000, 2001a.

120 Par exemple, Albero, 1998, 2001b ; Poteaux, 2000, 2003 ; Abé, 2003. 
distance (FOAD) ${ }^{121}$ débouchent davantage sur des recherches qui tendent à couvrir un champ de pratiques ${ }^{122}$ associant l'ingénierie de la formation ${ }^{123}$ et l'auto-direction ${ }^{124}$ des apprentissages. En tenant compte de l'environnement de l'acte d'apprendre, sans en disjoindre les éléments constitutifs ${ }^{125}$, ces travaux relativisent l'importance des moyens techniques. Ils les intègrent dans un système d'interactions où l'artefact s'inscrit dans un ensemble plus vaste, socialisé et institué. La limite de cette approche par les pratiques, c'est le pan d'impensé qu'elle laisse concernant les cultures, les systèmes de représentations, les savoirs et savoir-faire à l'œuvre dans l'activité instrumentée des formateurs et des apprenants. Il y a là, pour les sciences de l'éducation, un espace de recherche spécifique à déployer.

3.3. Une approche transversale et interdisciplinaire des problématiques et des questions de recherche

multiples niveaux (institutionnels, organisationnels, interpersonnels, individuels).

La complexité des phénomènes devient telle qu'il est difficile de les approcher selon des perspectives exclusivement monodisciplinaires et des méthodologies exclusives qu'elles soient ou non expérimentales. Si ces perspectives demeurent importantes en permettant des vérifications fines et armées d'hypothèses précises, l'apport des perspectives interdisciplinaires, voire transdisciplinaires, a son utilité également. C'est ainsi qu'après avoir réalisé plusieurs travaux de type expérimental (1973, 1984), M. Linard (1989, 2001) argumente en faveur des théories de l'activité, comme cadre organisateur plus large, source de références et de méthodologies différenciées mais cohérentes. Aujourd'hui, des recherches ${ }^{127}$ inspirées par la psychologie du travail (Clot,

${ }^{121}$ Par exemple, Jezegou, 1998. Revoir également la partie 1.2.4 de cette note.

122 Nous reprenons ici une analyse que fait J.-M. Barbier (2001) pour se référer aux recherches conduites sur les terrains des pratiques professionnelles.

123 Carré, Caspar, 1999.

124 Par exemple, Carré, 1993, 1995 ; Holec, 1994.

125 Par exemple, apprenant(s), formateur(s), contenu(s), moyen(s) technique(s), espace(s) de travail, etc.

126 Voir note 62.

127 Par exemple, Leblanc et al., 2001, 2003. 
1995, 2000) et par l'approche ergonomique (Theureau, 1992 ; Leplat, 1997), vont dans ce sens. Deux pistes apparaissent fécondes : la conceptualisation du terme d' «instrument» comme interface cognitive (Rabardel, 1995) et les travaux épistémologiques et méthodologiques sur les théories de l'action en éducation (Baudouin, Friedrich, 2001) et sur l'analyse de l'activité en formation (Barbier, 1996 ; Barbier, Durand, 2003 ; Durand et al., à paraitre).

La définition de l'instrument comme entité mixte sujet-objet, constitué à la fois d'un artefact et des représentations des utilisateurs, permet d'échapper à la focalisation sur les seuls moyens techniques en déplaçant l'analyse sur l'activité des acteurs et leurs usages. Cette activité est alors conçue comme un espace d'interaction entre instances de formation et d'apprentissage, dans le cadre d'une «instrumentation» technique, cognitive et sociale des apprentissages (Albero, 2004).

\subsection{Une prise en compte des perspec- tives axiologiques et praxéologiques} de formation, selon les termes de J.-M. Barbier (2001). D'un point de vue scientifique, un cadre d'analyse cohérent articulé à une méthodologie adaptée est indispensable. Mais ce point de vue n'évacue pas pour autant les autres dimensions de l'action de formation. En sciences de l'éducation, le chercheur se trouve toujours confronté à l'articulation d'une triple visée - «le vrai», «le juste ", «l'efficace» (Develay, 2001) - à partir des trois dimensions propres à son champ : scientifique, "axiologique », "praxéologique» (Meirieu, 1990). Encore faut-il en tirer les conséquences.

Si le chercheur ne souhaite pas se couper des acteurs qu'il observe, il est amené à penser ensemble ces trois dimensions d'une manière à la fois réflexive et critique. Il est également conduit à travailler constamment dans le mouvement de ses propres interactions avec les terrains (politiques et éducatifs) et de ses interrogations épistémiques et méthodologiques, que ce soit au niveau des terrains de la recherche, des conditions d'organisation et de déroulement de son activité, de l'articulation entre recherche et formation.

Sur les terrains, la prise en compte de la dimension axiologique et de l'intention d'optimisation des dispositifs de formation replace la recherche dans son contexte et nuance fortement les catégories et les modèles dégagés. Ce choix a des incidences multiples sur les critères d'analyse, les méthodologies 
déployées, la place accordée aux acteurs dans la démarche de recherche, les choix de formalisation et de présentation des résultats.

Pour le chercheur, le travail de rationalisation est à conduire dans la prise en compte réflexive des systèmes de valeurs et des intentions qui l'orientent. Cela entraîne le cadre théorique à se réorganiser à l'intérieur de paradigmes plus « compréhensifs» tels que le socio-constructivisme, la cognition distribuée et l'action située. Il en est de même des méthodologies de recueil de données qui ne peuvent que privilégier la mise en complémentarité des méthodes, plutôt que leur opposition. De même, les modes d'analyse des données sont-ils amenés à formaliser les résultats plutôt en termes de "dominantes", de "grandes tendances", inscrites dans des temporalités et perçues dans des cadres dynamiques qui produisent davantage des «configurations» que des états réifiés. Il s'ensuit également que la restitution des résultats sera à concevoir différemment selon le public auquel elle s'adresse (politiques, chercheurs du domaine, chercheurs dans d'autres domaines, acteurs éducatifs).

Enfin, la prise en compte de la dimension axiologique et praxéologique a une incidence sur l'articulation entre activité de recherche et activité de formation. Cette articulation est posée comme constitutive dans une tension entre les deux fonctions évoquées plus haut : l'apport d'éléments conceptuels de compréhension des phénomènes en jeu (intelligibilité) ; l'apport d'outils référentiels, conceptuels, méthodologiques et pratiques qui permettent aux acteurs de faire évoluer par eux-mêmes les situations vécues (savoirs, représentations, affects, savoir-faire, etc.) (optimisation).

\section{Pour conclure provisoirement}

Faire le point sur la recherche dans le domaine des technologies et de la formation fait surgir une métaphore maritime. Face à l'océan des travaux existants, quelle route peut-on tracer à partir de l'espace institutionnel des sciences de l'éducation, dans une époque de constante innovation technique, marquée par l'intrication d'intérêts économiques, de positionnements politiques et de fortes problématiques sociales?

Si le navigateur n'est pas encore un naufragé qui se laisse aller au gré des vents et des courants, faire le point est un exercice salutaire qui peut lui permettre de repérer à quel endroit il se trouve et comment il va manœuvrer pour prendre le chemin d'une destination qu'il se fixe.

La carte dressée dans cette note de synthèse peut paraittre grossière. Elle n’a d'autre ambition que de permettre une représentation globale d'un vaste territoire et l'élaboration par d'autres de cartes plus fines, sans oublier que, dans 
Note de synthèse - Technologies et formation

ce domaine pas plus qu'ailleurs, «la carte n'est pas le territoire » (Korzybski, 1933).

\section{Références}

ABÉ-HILDENBRAND D., 2003, «Autoformation des adultes et contexte institutionnel ", dans B. Albero, Autoformation et enseignement supérieur, Paris, Hermès/Lavoisier, pp. 141-152.

ADAMS D.M., HAMM M.E., 1990, Cooperative learning : Critical thinking and collaborations across the curriculum, Springfield, III, C.; C. Thomas.

AGNEL J. (dir.), 1994, Formations owvertes et à distance : la situation en France. Étude réalisée par la Commission des Communautés européennes, Paris, ORAVEP.

AIKEN R.M., EPSTEIN R.G., 2000, «Lignes directrices pour une éthique de l'utilisation des systèmes d'intelligence artificielle en éducation : éléments préliminaires pour un débat », Sciences et Techniques Éducatives, vol. 7, n 1, pp. 245265.

ALBERO B., 2004, L'instrumentation des apprentissages dans les dispositifs à finalité de formation des personnes, Note de synthèse pour l'habilitation à diriger des recherches, Paris 7 Denis Diderot.

ALBERO B., 2001a, «Pratiques d'apprentissage dans et hors institution : une dialectique enfin possible dans les dispositifs émergents de formation », Recherches en communication, $\mathrm{n}^{\circ} 15$, pp. 103-119.

ALBERO B., 2001b, «Les Espaces Langues : un potentiel d'évolutions des pratiques d'enseignement et des pratiques d'apprentissage ", Langues Modernes, $\mathrm{n}^{\circ} 2$, avrilmai-juin, pp. 76-84.

ALBERO B., 1998, 2000, L'autoformation en contexte institutionnel: du paradigme de l'instruction au paradigme de l'autonomie, Paris, L'Harmattan, Coll. Éducation et formation, Série Références, $306 \mathrm{p}$.

ALBERO B., 1998, «Le centre de ressources langues : interfaces entre matérialité et virtualité », Études de Lingnistique Appliquée, n 112, octobre-décembre, pp. 469-482.

ALTET M., BRITTEN J.D., 1983, Micro-enseignement et formation des enseignants, Paris, PUF, coll. Pédagogies d'aujourd'hui.

ANNOOT E., 2004, «Le tutorat ou le sens égaré », dans E. Annot, M.-F. Fave Bonnet (coord.), Les pratiques pédagogiques dans l'enseignement supérieur: enseigner, apprendre, évaluer, Paris, L'Harmattan, coll. Savoir et Formation, pp. 183-206.

ANNOOT E., 2001, « Le tutorat ou le temps suspendu », Revue des Sciences de l'Éducation, numéro thématique, vol. XXVII, nº 2, pp. 383-402.

ANNOOT E., 1998, "Tutorat et ressources éducatives : la question étudiante ", Perspectives Documentaires en Éducation, juin, pp. 59-72.

ANNOOT E., 1996, Les formateurs face aux nowvelles technologies : le sens du changement, Paris, Ophrys.

ARGYRIS C., 1970, Intervention theory and method, Reading, MA, Addison-Wesley.

ARGYRIS C., SCHÖN D. A., 1974, Theory in practice, San Francisco, Jossey-Bass. 
ARSAC J., 1987, Les machines à penser, Paris, Seuil.

AUDOUIN F., 1971, La pédagogie assistée. Cybernétique et enseignement, Paris, ESF.

AVEROUS M., TOUZOT G. (dir.), 2002, Campus numériques, enjeux et perspectives pour la formation ouverte et à distance, Rapport de mission, Paris, CNED. Disponible sur le site : http://www.educnet.education.fr

AZEMARD G., 1980, La vidéo, l'enfant et les institutions, Paris, Anthropos.

BALACHEFF N., 2001, « $\grave{A}$ propos de la recherche sur les environnements informatiques pour l'apprentissage humain », séminaire Cognitique, «Technologies de l'apprentissage ", Poitiers, 21 juin, http://wwwdidactique.imag.fr/Balacheff/TextesDivers/CognitiqueEIAH.html

BAPTISTE A., BEELISLE C., 1978, Photo-méthodes : comment utiliser Photolangage dans un travail de groupe, Paris, Chalet.

BARBARANT J.-C., 1997, Enseignement à distance : réalités, enjeux et perspectives. Rapport présenté au nom de la section des Affaires sociales, Paris, Conseil économique et social.

BARBIER J.-M., 2001, «La constitution de champs de pratiques en champs de recherches ", dans J.-M. Baudouin et J. Friedrich (eds), Théories de l'action et éducation, Bruxelles, De Boeck Université, pp. 305-317.

BARBIER J.-M., 2000, «Sémantique de l'action et sémantique de l'intelligibilité des actions. Le cas de la formation ", dans B. Maggi (sous la dir. de), Manières de penser, manières d'agir en éducation et en formation, Paris, PUF, coll. Éducation et formation, pp. 89-104.

BARBIER J.-M. (dir.), 1996, Savoirs théoriques et savoirs d'action, Paris, PUF, coll. Pédagogies d'aujourd'hui.

BARBIER J.-M., DURAND M. (coord.), «L'analyse de l'activité. Approches situées », Recherche et formation pour les professions de l'éducation, $\mathrm{n}^{\circ} 42$.

BARON G.-L., 2001, «L'institution scolaire confrontée aux TIC», Sciences bumaines, $\mathrm{n}^{\circ}$ 32, mars-avril-mai, pp. 48-51.

BARON G.-L., 1989, L’informatique, discipline scolaire ?, Paris, PUF, coll. Pédagogie d'aujourd'hui.

BARON G.-L., BRUILLARD E., 1996, L'informatique et ses usagers dans l'éducation, Paris, PUF, coll. L'éducateur.

BARON G.-L., BAUDÉ J. (eds), L'intégration de l'informatique dans l'enseignement et la formation des enseignants, Paris, INRP.

BARON G.-L., BAUDÉ J., LA PASSARDIÈRE B. de, 1993, Hypermédias et apprentissages. Actes des deuxièmes journées scientifiques, 24-25 mars, Lille, EPI/CUEPP/INRP.

BATES A.W. (ed.), 1990, Media and Technology in European Distance Education. Proceedings of the EADTU workshop on Media, Methods and Technology, Herleen, European Association of Distance Teaching Universities.

BATES A.W., 1984, The Role of Technology in Distance Education, Londres, Croom Helm/New York, St Martin's Press. 
Note de synthèse - Technologies et formation

BAUDOUIN J.-M., FRIEDRICH J. (eds), 2001, Théories de l'action et éducation, Bruxelles, De Boeck Université.

BAUDRILLARD J., 1968, Le système des objets, Paris, Gallimard, coll. Tel., nº 33.

BEILLEROT J., 1982, La société pédagogique, Paris, PUF.

BÉRANGER SERT D., 1981, Contribution à l'analyse d'une technique de communication : le cas de la lanterne magique à la fin du XIX ${ }^{\text {ime }}$, son utilisation dans la formation des adultes : éléments pour l'élaboration d'une cartographie, Thèse de doctorat, Paris, EHESS.

BERNARD M., 1999, Penser la mise à distance en formation, Paris, L'Harmattan, coll. Éducation et formation, série Références.

BERTRAND J.-L., CORSET P., MARMOUD D., 1986, Utilisation du réseau câblé dans le secteur de la formation professionnelle, Paris, INA, Écully, CEREP.

BIREAUD A., 1982, Systèmes d'autoformation : étude historique d'un événement pédagogique : le centre d'autodocumentation du CES de Marly-le-Roi, Thèse d'État, Université Paris 13.

BIREAUD A., 1979, Le collège audiovisuel de Marly-le-Roi. Une innovation en technologie éducative : 1963-1970, étude historique, Thèse de troisième cycle, Université Paris 13.

BLANDIN B., 2002, La construction du social par les objets, Paris, PUF.

BLANDIN B., 1990, Formateurs et formation multimédia, Paris, éd. d'Organisation.

BOULLIER D., 2001, "Les choix techniques sont des choix pédagogiques : les dimensions multiples d'une expérience de formation à distance ", Sciences et techniques éducatives, vol. 8, $\mathrm{n}^{\circ} 3-4, \mathrm{pp} .275-299$.

BOULLIER D., 1997, «Des interfaces à l'homme. Assurer l'empreinte pédagogique sur les NTE », Dossiers de l'audiovisuel, sept.-oct., pp. 66-68.

BOUGNOUX D., 1993, Sciences de l'information et de la communication, Paris, Larousse, coll. Textes essentiels.

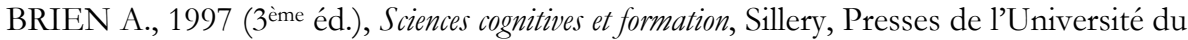
Québec.

BRUILLARD E., 1997, Les machines à enseigner, Paris, Hermès.

BRUILLARD E., DE LA PASSARDIÈRE B., 1994, «Hypermédias et éducation : des repères ", Sciences et techniques éducatives, $\mathrm{n}^{\circ} 1$, pp. 17-38.

BRUNSWIC E., 1970, «Hier l'audiovisuel, demain la technologie de l'éducation », Média, $\mathrm{n}^{\circ} 18$, décembre, pp. 15-22.

BUCHER-POTEAUX N., 1997, «L'intégration des nouvelles technologies éducatives dans l'enseignement des langues ", dans M-C. Cuellar (ed), La nuevas technologias integradas en la programacion didactica de lenguas extranjeras, I Encuentro Internacional Universidad Internacional Menendez Pelayo, pp.19-28.

CALLON M., 1998, "Défense et illustration des recherches sur la science ", dans B. Jurdant (sous la dir. de), Impostures scientifiques. Les malentendus de l'affaire Sokal, Paris, La Découverte Alliage, pp. 253-267.

CARRÉ P., 1997, «L'individualisation de la formation : une technologie au service de l'autoformation?", Les Cabiers d'études du CUEPP.

CARRÉ P., 1995, «L'autodirection en formation. Contribution à l'analyse ternaire de l'autoformation », Éducation permanente, n 122, pp. 221-232. 
Savoirs, 5, 2004

CARRÉ P., 1993, L'autodirection en formation, Note de synthèse pour l'habilitation à diriger des recherches, Tours, Université François Rabelais.

CARRÉ P., 1992, L'autoformation dans la formation professionnelle, Paris, La Documentation Française, Coll. Recherche en formation continue.

CARRÉ P., 1991, Organiser l'apprentissage des langues étrangères. La formation linguistique professionnelle, Paris, éd. d'Organisation.

CARRÉ P., MOISAN A. (eds.), La formation autodirigée: aspects psychologiques et pédagogiques, Paris, L'Harmattan.

CARRÉ P., CASPAR P. (eds), 1999, Traité des sciences et des techniques de la formation, Paris, Dunod.

CARRÉ P., MOISAN A., POISSON D., 1997, L'autoformation, Paris, PUF, coll. Pédagogies d'aujourd'hui.

CARRÉ P., CLENET J., D’HALLUIN C., POISSON D., 1999, «Ingénierie pédagogique et formations ouvertes », dans P. CARRÉ, P. CASPAR (eds), Traité des sciences et des techniques de la formation, Paris, Dunod.

CASPAR P. (dir.), 1991, Le savoir à portée de la main. La conduite de projets de formation multimédia, Paris, éd. d'Organisation.

CASTORIADIS C., 1978, Les carrefours du labyrinthe 1, Paris, Seuil.

CASTORIADIS C., 1975, «Technique », Encyclopadia Universalis, vol. 15, pp. 803-809.

CHALVON M., CORSET P., SOUCHON M., 1979, L'enfant devant la télévision, Paris, Casterman.

CHANIAC R., 1975, Neuf expériences de la télévision éducative dans le monde, Bry-sur-Marne, INAP.

CHANIER T., POTHIER M. (dir.), 1998, « Hypermédia et apprentissage des langues », Études de linguistique appliquée, $\mathrm{n}^{\circ} 110$.

CHAPTAL A., 1993, «Les habits neufs du multimédia », Médiaspouvoirs, n 31-32, pp. 203-212.

CHARLIER B., PERAYA D., 2003, Technologie et innovation en pédagogie. Dispositifs innovants de formation pour l'enseignement supérieur, Bruxelles, De Boeck.

CHARLOT B., 1995, Les sciences de l'éducation, un enjeu, un défi, Paris, ESF, coll. Pédagogies.

CHOPLIN H. (coord.), 2002, «Les TIC au service des nouveaux dispositifs de formation ", Éducation Permanente, $\mathrm{n}^{\circ} 152$.

CLERGUE G., 1997, L'apprentissage de la complexité, Paris, Hermès.

CLOT Y., 2000, "Analyse psychologique du travail et singularité de l'action », dans J.M Barbier (dir.), L'analyse de la singularité de l'action, Paris, PUF, coll. Éducation et formation, pp. 53-69.

CLOT Y., 1999, La fonction psychologique du travail, Paris, PUF, coll. Le travail humain.

CLOT Y., 1995, "Qu'est-ce que l'activité dans l'analyse du travail ? ", Performances bumaines et techniques, hors série «À quoi sert l'analyse de l'activité en ergonomie ?».

COLEMAN D., FUREY D., 1996, «Collaborative Infrastructure for Knowledge Management », http:// collaborate.com 
Note de synthèse - Technologies et formation

COMPTE C., 2003, «Images et apprentissages : une médiation pour l'autoformation », dans B. Albero (dir.), Autoformation et enseignement supérieur, Paris, Hermès/Lavoisier, pp. 219-239.

COMPTE C., 2001, «Écriture Télévisuelle et médiation cognitive ou comment la télévision véhicule des savoirs formels et informels ", Recherches en Communication, $\mathrm{n}^{\circ}$ 16, Interfaces sémiotiques et cognition, Université Catholique de Louvain, pp. 131 152.

COMPTE C., 1999, «Introduction des nouvelles technologies dans la transmission des connaissances, la mise en place de dispositifs de formation des maitres, problématiques et réflexion ", Troisième Congrès International d'Actualité de la Recherche en Éducation et Formation, juin, AECSE, Université de Bordeaux III, CDRom.

COMPTE C., 1998, «La rhétorique télévisuelle : une médiation pour l'apprentissage des langues », Les Cabiers du CIRCAV (Centre Interdisciplinaire de Recherche sur les Communications Audiovisuelles), $\mathrm{n}^{\circ}$ 10, Université de Lille III, Gerico, pp. 227-249.

COMPTE C., 1995, Exploitation didactique de l'image animée, Habilitation à Diriger des Recherches, Université Paris 7 - Denis Diderot.

COMPTE C., 1993, La vidéo en classe de langue, Paris, Hachette, coll. F.

COMPTE C., 1985a, Using Soap Opera Structure for Aural French Comprehension, PhD, New York University.

COMPTE C., 1985b, « Professeur cherche document authentique en vidéo », Études de Linguistique Appliquée, $\mathrm{n}^{\circ}$ 58, pp. 43-53.

COMPTE C., 1985c, Où en est le vidéodisque de langues ?, Paris, BELC/CIEP.

COMPTE C., 1984, «Spécificité télévisuelle et enseignement du Français langue étrangère : articulation des objectifs de la classe de langue et de l'écriture télévisuelle », dans Approches communicatives. Vidéo et télévision dans l'enseignement des langues, Instituto de Ciencias de la educación, universidad autónoma de Barcelona, pp. $83-107$.

Conférence des Présidents d'université (CPU), 1999, Impact des nouvelles technologies sur les acteurs de l'université, Actes des entretiens de la Conférence réunie à Nancy, le 18 mars 1999, Paris, CPU. Disponible sur le site : http://www.cpu.fr/Telecharger/Actes_Nancy.pdf

CRINON J., GAUTELLIER C., 1997, Apprendre avec le multimédia : où en est-on ?, Paris, Retz.

DECOTE G., 1967, Vers l'enseignement programmé, Paris, Gauthier-Villars.

DEJOURS C., 1998, Souffrance en France. La banalisation de l'injustice sociale, Paris, Seuil.

DEKEN J., 1983, 1984, Les images du futur, Paris, éd. Mazarine.

DELAVILLE A., 1986, Dix années de techniques audiovisuelles dans l'enseignement 1976-1985, Paris, CNDP, coll. Références documentaires.

DE LIÈVRE B., DEPOVER C., 1999, "Analyse des processus d'apprentissage dans une situation de tutorat à distance », dans C. Depover, B. Noël (eds), L'évaluation des compétences et des processus cognitifs. Modèles, pratiques et contextes, Bruxelles, De Boeck/Larcier, pp. 283-302. 
DEMAIZIÈRE F., 1986, Enseignement assisté par ordinateur, Paris, Ophrys.

DEMAIZIÈRE F., DUBUISSON C., 1992, De l'EAO aux NTF. Utiliser l'ordinateur pour la formation, Paris, Ophrys.

DEPOVER C., 1987, L'ordinateur média d'enseignement : un cadre conceptuel, Bruxelles, De Boeck-Wesmael.

DEPOVER C., GIARDINA M., MARTON P., 1996, Les environnements d'apprentissage multimédia. Analyse et conception, Paris, L'Harmattan, coll. Éducation et formation, série Références.

DERYCKE A., HOOGSTOEL F., VIÉVILLE C., 1997, "Campus virtuel et apprentissage coopératif », dans Actes des cinquièmes journées ELAO AFCET, Cachan, 14-16 mai, Paris, Hermès, pp. 11- 24.

DESMOULINS C., MARQUET P., BOUHINEAU D., 2003, Environnements informatiques pour l'apprentissage bumain. Actes de la conférence ELAH 2003, Strasbourg, 15, 16 et 17 avril, ATIEF/INRP.

DESPRÈS C., LEROUX P., 2003, «Tutorat synchrone en formation à distance », dans C. Desmoulins, P. Marquet, D. Bouhineau, Environnements informatiques pour l'apprentissage bumain. Actes de la conférence ELAH 2003, ATIEF/INRP, pp. 139-150.

DEVELAY M., 2001, Propos sur les sciences de l'éducation : réflexions épistémologiques, Paris, ESF.

D'HALLUIN C. (dir.), 2001, «Usages d'un environnement médiatisé pour l'apprentissage coopératif », Cabiers du CUEEP, n 43.

D’HALLUIN C. (coord.), 1993, « Une pratique d'enseignement ouvert. La préparation de l'ESEU en enseignement à distance en 1991-1992 ", Les Cabiers du CUEPP, n 22.

D'HALLUIN C., HAEUW F. (coord.), 1995, « Formations ouvertes multiressources. Actes de l'université d'été, Lille, 6-12 juillet 1994 », Les Cabiers du CUEPP, nº 28.

DIEUZEIDE H., 1985, «Enjeux politiques de la formation à distance », dans F. Henri, A. Kaye, Le savoir à domicile : pédagogie et problématique de la formation à distance, Québec, Presses de l'Université, pp. 29-61.

DIEUZEIDE H., 1974, «La radio-télévision au service de l'éducation en Europe occidentale. Quelques remarques sur son état présent et ses perspectives », Revue de l'UER, vol. XXV, n ${ }^{\circ}$ 2, mars, pp. 12-20.

DIEUZEIDE H., 1970, Technologie éducative et développement de l'éducation, Paris, UNESCO, coll. Année internationale de l'éducation, $\mathrm{n}^{\circ} 8$.

DIEUZEIDE H. 1965, Les techniques audio-visuelles dans l'enseignement, Paris, PUF.

DILLENBOURG P., 2000, «Virtual Learning Environments», http://tecfa.unige.ch/tecfa/publicat/

DON D., 1988, Dictionnaire de l'E $A O$, Paris, Ophrys.

DUCASSÉ P., 1958, Les Techniques et le philosophe, Paris, PUF.

DUHAMEL O., 1990, Pour une université ouverte. Conclusions à M. Lionel Jospin, Paris, rapport multigraphié.

DUPLÀA E., GALISSON A, CHOPLIN H., 2003, « Le tutorat à distance existe-t-il ? Propositions pour du tutorat proactif à partir de deux expérimentations de 
FOAD », dans C. Desmoulins, P. Marquet, D. Bouhineau, Environnements informatiques pour l'apprentissage bumain. Actes de la conférence ELAH 2003, ATIEF/INRP, pp. 477-484.

DURAND M. VEYRUNES P., CHALIÈS S. (à paraître), "L'analyse de l'activité en situation : questions de méthodes », Éducation Permanente.

EGLY M., 1984, Télévision didactique, entre le kitsch et les systèmes du troisième type, Paris, Edilig, coll. Médiathèque.

EGLY M., 1981, L'impact de la télévision éducative sur les jeunes enfants, Paris, UNESCO, coll. Études de documents d'éducation.

ELLUL J., 1988, Le bluff technologique, Paris, Hachette.

ELLUL J., 1977, Le système technicien, Paris, Calmann-Lévy.

ELLUL J., 1954, La Technique ou l'Enjeu du siècle, Paris, Armand Colin.

EHRLICH K., CASH D., 1996, «Turning information into knowledge : Information finding as a collaborative activity », http://www-cscl95/indiana.edu/cscl95.html

FAUQUET G., STRASVOGEL S., 1972, L'audiovisuel an service de la formation des enseignants, le circuit fermé de télévision, Paris, Delagrave.

FEENBERG A., 1999, 2004, (Re)penser la technique. Vers une technologie démocratique, trad. par A.-M. Dibon (Questioning Technology), Paris, La Découverte, coll. Recherches.

FICHEZ E. (coord.), 1993, «Éducation et formation. Le temps de l'industrialisation ? ", Études de communication, $\mathrm{n}^{\circ} 14$.

FILLOUX J.-C., 2001, Épistémologie, éthique et sciences de l'éducation, Paris, L'Harmattan, coll. Éducation et philosophie.

FINKIELKRAUT A., SORIANO P., 2001, Internet : l'inquiétante extase, Fondation du 2 mars, Mille et Une nuits.

FLAGEUL A., 1974, Télévision et éducation des adultes en France, Paris, Service de la recherche de l'ORTF.

FLICHY P., 1995a, L’innovation technique. Récents développements en sciences sociales. Vers une nouvelle théorie de l'innovation, Paris, La Découverte.

FLICHY P., 1995b, "L'action dans un cadre socio-technique. Comment articuler techniques et usage dans une même analyse », dans J.-G. Lacroix, G. Tremblay, Les autoroutes de l'information. Un produit de la convergence, Montréal, Presses Universitaires du Québec, pp. 405-433.

FLICHY P., 1980, 1991, Les industries de l'imaginaire. Pour une analyse économique des médias, Grenoble, PUG.

FREICHE J., 1972, «Le magnétoscope et l'image du corps », Éducation permanente, $\mathrm{n}^{\circ}$ 14, pp. 23-36.

FRIEDMANN G., 1966, «L'école parallèle », Le Monde, 7-12 janvier.

GARDNER H., 1985, 1993, Histoire de la révolution cognitive. La nouvelle science de l'esprit, trad. par J.-L. Peytavin (The mind's new science. A history of the cognitive revolution), Paris, Payot.

GAVINI G., 1965, Manuel de formation aux techniques de l'enseignement programmé, Paris, éd. Hommes et techniques. 
GERMAIN G., GABRIEL M., 1985, Le vidéodisque, banque d'images interactives, Paris, Cédic-Nathan.

GIARDINA M., 1999, L'interactivité, le multimédia et l'apprentissage, Paris, L'Harmattan, coll. Éducation et formation, série Références.

GILLE B., 1978, Histoire des techniques, Paris, Gallimard, coll. Encyclopédie de la Pléiade.

GLIKMAN V., 2002, Des cours par correspondance au «e-learning», Paris, PUF, coll. Éducation et formation.

GLIKMAN V. (éd.), 1999, Formations ouvertes et à distance : le point de vue des usagers, Journée d'étude du 28 novembre 1997, Paris, INRP, Technologie nouvelles et éducation.

GLIKMAN V., 1989, Évolution d'une politique en matière de technologie éducative : histoire de RTS Promotion, une expérience française de télévision éducative pour adultes (1964-1985), Thèse pour le doctorat de sciences de l'éducation, Université Paris V - René Descartes.

GLIKMAN V., BARON G.-L., 1991, «Médias, multi-médias, technologies et formation à distance. Quelques éléments pour l'histoire d'un concept et une analyse de sa réalité au début des années quatre-vingt-dix », Perspectives documentaires en éducation, $\mathrm{n}^{\circ} 24$, pp. 63-93.

GLIKMAN V., CHRETIEN C., 1991, Bilan des usages français du satellite éducatif Olympus en 1990 et principales caractéristiques des programmes, Paris, INRP/IFACE/ATENA.

GRANDBASTIEN M., 1989, 1990, Les technologies nouvelles dans l'enseignement général et technique : situation au terme des années 80 et propositions d'orientations pour la décennie à venir, Paris, La Documentation française, coll. Rapports officiels.

GUIHOT P., 1993, «Communication par voie télématique à l'école », dans Le point sur les recherches achevées à l'INRP en 1991 et 1992, Paris, INRP.

GUILLERME J., 1975, «Technologie », Encyclopadia Universalis, vol. 15, pp. 820-823.

GUZDIAL M., TURNS J. RAPPIN N. CARLSON D., 1995, «Collaborative support for learning in complex domains », http:/ /www-cscl95/indian.edu/cscl95/

HABERMAS J., 1968, 1973, La technique et la science comme "idéologie », trad. par J.-P. Ladmiral (Technik und wissenschaft als ideologie), Paris, Gallimard, coll. Tel.

HARASIM L., 1990, Online education. Perspectives on a new environnement, New York, Prager.

HAUDRICOURT A.-G., 1987, La technologie science bumaine. Recherches d'bistoire et d'ethnologie des techniques (1936-1978), Paris, éd. de la Maison des sciences de l'homme.

HEIDEGGER M., 1954, 1958, «La question de la technique », Essais et conférences, trad. par A. Préau (Vorträge und aufsätze), Paris, Galimmard, coll. Tel, pp. 9-48.

HENRI F., LUNDGREN-CAYROL K., 2001, Apprentissage collaboratif à distance. Pour comprendre et concevoir les environnements d'apprentissage virtuels, Sainte-Foy, Presses de l'Université du Québec.

HENRI F., KAYE A., 1985, Le savoir à domicile : pédagogie et problématique de la formation à distance, Québec, Presses de l'Université. 
HOLEC H., 1994, L'Apprentissage Auto-Dirigé : une autre offre de formation. Principes, implications, réalisations, Strasbourg, Conseil de la Coopération Culturelle du Conseil de l'Europe.

HOLTZ-BONNEAU F., 1986, L'image et l'ordinateur, Paris, Aubier, INA.

HORKHEIMER M., ADORNO T. W., 1974, 1983, «La production industrielle des biens culturels ", dans La dialectique de la raison: Fragments philosophiques, trad. par E. Kaufholz (Dialectik der Aufklärulg : philosophische Fragmente), Paris, Gallimard.

HOTTE R., 1995, L'intervention tutorale dans un système d'encadrement pédagogique assisté par ordinateur (EPAO), Québec, Télé-Université.

HOTTOIS G., 2001, "Y-a-t-il une philosophie de la technique ? ", dans T. Ferenczi, Penser la technique, Bruxelles, éd. Complexe, coll. Poche, pp. 27-45.

HOTTOIS G., 1984, Le Signe et la technique (la philosophie à l'épreuve de la technique), Paris, Aubier-Flammarion.

HUET A., ION J., LEFEBVRE A., MIÈGE B., PERON R., 1978, 1984 (2ème éd.), Capitalisme et industries culturelles, Grenoble, PUG.

ISAMBERT-JAMATI V., 1984, Culture technique et culture sociale à l'école élémentaire, Paris, PUF.

JACQUINOT G., 1993, «Apprivoiser la distance et supprimer l'absence ? ou les défis de la formation à distance », Revue française de pédagogie, $\mathrm{n}^{\circ} 102$, pp. 55-67.

JACQUINOT G., 1985, L'école devant les écrans, Paris, ESF, coll. Sciences de l'éducation.

JACQUINOT G., 1977, Image et pédagogie : analyse sémiologique du film à intention didactique, Paris, PUF, coll. L'éducateur.

JACQUINOT-DELAUNAY G., MONNOYER L. (coord.), 1999, Le dispositif. Entre usage et concept, Hermès, $\mathrm{n}^{\circ} 25$, Paris, CNRS éd.

JÉZÉGOU A., 1998, La formation à distance : enjeux, perspectives et limites de l'individualisation, Paris, L'Harmattan.

JULLIEN F., 1996, Traité de l'efficacité, Paris, Le livre de poche, coll. Biblio essais, ${ }^{\circ}$ 4292.

JONAS H., 1993, 1998, Pour une éthique du futur, trad. par S. Cornille et P. Ivernel (Philosophie. Rückschau und Vorschau am Ende des Jahrhunderts), Paris, Payot \& Rivages.

JONAS H., 1979, 1990, Le principe de responsabilité : une éthique pour la civilisation technologique, trad. par J. Greisch (Das Prinzip Verantwortung, Frankfurt, Verlag), Paris, Éd. du Cerf/Flammarion.

JURDANT B. (dir.), 1998, Impostures scientifiques. Les malentendus de l'affaire Sokal, Paris, La Découverte/Alliage.

KAGAN S., 1992, Cooperative learning, San Juan Capistrano, Ressources for Teachers.

KANSELAAR G., ERKENS G., 1996, A cooperative system for collaborative problem solving, Netherlands, Department of Educational Sciences, University of Ultrecht.

KARSENTI T., LAROSE F. (eds), 2001, Les TIC... au cœur des pédagogies universitaires, Sainte-Foy, Presses de l'Université du Québec.

KAYE A., 1988, «L'enseignement à distance : un état de la question », Prospective, vol. XVIII, n 1 , pp. 43-54. 
Savoirs, 5, 2004

KAYE A., RUMBLE G., 1981, Distance Teaching for Higher Adult Education, Londres, Croom Helm.

KAYSER D., 1987, « Raisonnement : logique et informatique », Intellectica, n 1, pp. 81103.

KEEGAN D. (ed.), 1993, Theorical principles of distance education, London, Routledge.

KITTLER F., 2002, "Médias de la communication", dans C. Wulf (dir.), Traité d'anthropologie historique. Philosophies histoires cultures, Paris, L'Harmattan, pp. 649-662.

KORZYBSKI A., 1933, 1980, Science and Sanity. The International Non-Aristotelician, Lakeville, Lib. Pub. Cy.

KUPERHOLC J., MOR A., 1991, «Les 'systèmes souples' de formation : leurs effets sur les mutations de l'appareil de formation », Études et expérimentations en formation continue, $\mathrm{n}^{\circ} 11$, pp. 17-20.

KUPERHOLC J., MOR A., PIETRE F., 1993, Développer de nouvelles formes de formation : les systèmes flexibles, Paris, Liaisons.

LA BORDERIE R., 1972, «La communication audio-visuelle, éléments pour une approche systématique », Éducation permanente, $\mathrm{n}^{\circ} 14$, pp. 3-20.

LACROIX C., PETIT M., ROUET F., 1979, Les industries culturelles, Paris, La documentation française.

LAMOUROUX G., MORE R., 1987, « Point de vue critique sur l'enseignement assisté par ordinateur », Bulletin de la Société Alfred Binet et Théodore Simon, n 612, pp. 16-21.

LA PASSARDIÈRE B. de, BARON G.-L., 1991, Hypermédias et apprentissages. Actes des premières journées scientifiques, 24-25 septembre, Paris, INRP.

LATOUR B., 1999, 2001, L'espoir de Pandore. Pour une version réaliste de l'activité scientifique, trad. Didier Gille (Pandora's Hope. Essays on the Reality of Sciences Studies), Paris, La Découverte.

LATOUR B., 1994, 2001 (2ème éd. revue et corrigée), Le métier de chercheur. Regard d'un anthropologue, Une conférence-débat à l'INRA, Paris, le 22 septembre 1994, Paris, INRA éditions, coll. Sciences en questions.

LEBEAUME J., 2000, L'éducation technologique. Histoires et Méthodes, Paris, ESF.

LEBEAUME J., MARTINAND J.-L. \& al., 1998, Enseigner la technologie au collège, Paris, Hachette.

LEBLANC S, SAURY J., SÈVE C., DURAND M., 2003, «Les interactions utilisateurs-environnement hypermédia en situation réelle de formation », Savoirs, $\mathrm{n}^{\circ} 3$, pp. 55-73.

LEBLANC S., SAURY J., SÈVE C., DURAND M., THEUREAU J., 2001, «An Analysis of a User's Exploration And Learning of a Multimedia Instruction System», Computers \& Education, n 36 , pp. 59-82.

LEBRUN M., 2002, Théories et méthodes pédagogiques pour enseigner et apprendre. Quelle place pour les TIC dans l'éducation?, Bruxelles, De Boeck Université.

LEBRUN M., VIGANÒ R., 1995, «De l' 'Educational Technology' à la technologie pour l'éducation ", Les cabiers de la recherche en éducation, $\mathrm{n}^{\circ} 2 / 3$, pp. 457-482.

LEHNISCH J.-P., 1980, Enseignement à distance et formation professionnelle continue, Paris, ESF. 
LEHNISCH J.-P., 1981, La formation à distance, Paris, PUF.

LEGROS D., CRINON J. (dir.), 2002, Psychologie des apprentissages multimédia, Paris, Armand Colin.

LEGROS D., CRINON J., GEORGET P., 2000, Les effets des systèmes et des outils multimédias sur la cognition, l'apprentissage et l'enseignement, Rapport du Comité national de coordination de la recherche en éducation (CNCRE), Paris, http://archiveedutice.ccsd.cnrs.fr/edutice-00000351

LEMAIRE B., MARQUET P., BAILLÉ J., 1997, «Étude comparative du discours de l'enseignant et du comportement des étudiants en situations de cours traditionnel et d'enseignement à distance informatisé », Carrefours de l'éducation, nº 3, pp. 75-91.

LE NY J.-F., RICHARD J.-F., 1986, « Intelligence artificielle, science cognitive », dans C. Bonnet, J.-M. Hoc, G. Tiberghien (eds), Intelligence artificielle automatique et psychologie, Bruxelles, Mardaga.

LEPLAT J., 2000, L'analyse psychologique de l'activité en ergonomie. Aperç sur son évolution, ses modèles et ses méthodes, Toulouse, Octarès.

LEPLAT J., 1997, Regards sur l'activité en situation de travail. Contribution à la psychologie ergonomique, Paris, PUF, coll. Le travail humain.

LEPLAT J., 1991, "Voies de recherche et champs d'intervention dans les nouvelles technologies ", Bulletin de psychologie, t. XLV, $\mathrm{n}^{\circ} 404$.

LERBET G., 1992, L'école du dedans, Paris, Hachette, coll. Éducation, Pédagogies pour demain, Références.

LEROI-GOURHAN A., 1964, 1965, Le geste et la parole. Technique et langage (t. 1), La mémoire et les rythmes (t. 2), Paris, Albin Michel.

LEROI-GOURHAN A., 1943, 1945, Évolution et technique. L'Homme et la matière (t.1), Milieu et techniques (t.2), Paris, Albin Michel.

LÉVY P., 2000, World Philosophy, Paris, Odile Jacob, coll. Le champ médiologique.

LÉVY P., 1990, 1992, Les technologies de l'intelligence. L'avenir de la pensée à l'ère informatique, Paris, La Découverte, coll. Sciences et société.

LÉVY P., 1987, La machine univers, Paris, La Découverte, coll. Sciences et société.

LINARD M., 2003, «Autoformation, éthique et technologies : enjeux et paradoxes de l'autonomie », dans B. Albero (dir.), Autoformation et enseignement supérieur, Paris, Hermès/Lavoisier, pp. 241-263.

LINARD M., 2001, "Concevoir des environnements pour apprendre: l'activité humaine, cadre organisateur de l'interactivité technique », dans E. Delozanne et P. Jacoboni, Sciences et techniques éducatives, vol. 8, n 3-4, pp. 211-238.

LINARD M., 1989, 1996 (nouv. éd. réactualisée.), Des machines et des hommes. Apprendre avec les nouvelles technologies, Paris, L'Harmattan, coll. Savoir et formation.

LINARD M., 1987, Machines à représenter. L'analogie des images et la logique de l'ordinateur, Thèse d'État, Université Paris X-Nanterre.

LINARD M., 1975, «Un jeu de déchiffrement de l'image : le photorécit», Communications et langages, $\mathrm{n}^{\circ} 28$, pp. 95-116.

LINARD M., 1973, Les effets du feed-back par télévision sur le processus enseigner-apprendre en situation de petits groupes, Thèse de Troisième cycle, Université Paris X-Nanterre. 
LINARD M., PRAX I., 1984, Images de soi on Narcisse an travail, Paris, Dunod.

LOCHARD J., 1995, La formation à distance ou la liberté d'apprendre, Paris, éd. d'Organisation.

LUNDGREN-CAYROL K., 1996, Computer-Conferencing : A collaborative learning environment for distance education students, Thèse de doctorat, Montréal, Université Concordia.

LURCAT E., 1984, Le jeune enfant devant les apparences télévisuelles, Paris, ESF.

LURCAT E., 1981, À cinq ans, seul avec Goldorak : le jeune enfant et la télévision, Paris, Syros, coll. Contre-poisons.

MANGENOT F., 1999, "L'intérêt pédagogique des mondes virtuels », dans J. Anis (ed.), Internet, Communication et Langue française, Paris, Hermès, pp. 93-111.

MANGENOT F., 1998, «Classification des apports d'Internet à l'apprentissage des langues ", Apprentissage des langues et Systèmes d'Information et de Communication (ALSIC), $\mathrm{n}^{\circ} 3$ (2), pp. 187-206, http://alsic.org

MARCUSE H., 1964, 1968, L'homme unidimensionnel, trad. de M. Wittig (One Dimensional Man. Studies in the Ideology of Advenced Industrial Society), Paris, éd. de Minuit, coll. Arguments.

MARIET F., 1989, Laissez-les regarder la télévision, Paris, Calmann-Lévy.

MARTINAND J.-L., 1995, «Rudiments d'épistémologie appliquée pour une discipline nouvelle : la technologie », dans M. Develay, Épistémologie des savoirs scolaires, Paris, ESF, pp. 339-352.

MARTINAND J.-L., 1994, La technologie dans l'enseignement général : les enjeux de la conception et de la mise en cuvre, UNESCO, IIPE.

MATTELART A., PIEMME J.-M., 1980, Télévision, enjeux sans frontières, industries culturelles et politique de communication, Grenoble, PUG.

MC LUHAN M., 1967, La galaxie Gutemberg face à l'ère électronique. Les civilisations de l'âge oral à l'imprimerie, trad. par J. Paré (The Gutemberg galaxy), Paris, Mame.

MEIRIEU P., 1990, «Le modèle et le pédagogue », Le groupe familial, n 129, pp. 6-10.

MIÈGE B., 1993, La pensée communicationnelle, Grenoble, PUG, coll. La communication en plus.

MIÈGE B., 1989, La société conquise par la communication, Grenoble, PUG.

MINSKY M., 1968, 1985, Semantic Information and Processing, Cambridge MA, MIT Press.

MOEGLIN P. (dir.), 1998, L'industrialisation de la formation. État de la question, Paris, CNDP, coll. Documents, actes et rapports pour l'éducation.

MOEGLIN P., 1993, «Le paradigme de la machine à enseigner», dans E. Fichez (coord.), "Éducation et formation. Le temps de l'industrialisation ?», Études de communication, $\mathrm{n}^{\circ} 14$, pp. 91-103. 
Note de synthèse - Technologies et formation

MOEGLIN P., 1988, Vers l'industrialisation des communications éducatives, scientifiques et communautaires : étude critique des programmes expérimentaux au Canada, en France et aux USA (1971-1981), Thèse de doctorat, Grenoble, Université Stendhal.

MORIN E., 1982, 1990, Science avec conscience, Paris, Fayard et Seuil, coll. Points Sciences, $\mathrm{n}^{\circ} \mathrm{S} 64$.

MONTMOLLIN M. de, 1965, 1971 (3ème éd.), L'enseignement programmé, Paris, PUF.

MOTTET G., 1997 (dir.), La vidéo-formation : autres regards, autres pratique, Paris, L'Harmattan.

MOTTET G., MAIRE S. (coord.), 1975, 1979, Formation des maîtres, Bulletin de Liaison des Comité de Coordination des Écoles Normales, $\mathrm{n}^{\circ} 1$ et 2, Paris, OFRATEME, $\mathrm{n}^{\circ} 3$ à 5 , Paris, CNDP.

MUCCHIELLI A., 1987, L'enseignement par ordinateur, Paris, PUF.

MUMFORD L., 1967, 1973, Le mythe de la machine, 2 vol., trad. par L. Dilé (The Myth of the Machine), Paris, Fayard, coll. Le phénomène scientifique.

MUMFORD L., 1934, 1950, Technique et civilisation, trad. par D. Moutonnier (Tecnics and Civilization), Paris, Seuil.

NANARD M., 1994, "L'apport des travaux de recherche dans les hypertextes aux techniques éducatives ", dans E. Bruillard et al. (eds), Actes du séminaire Hypermédias, éducation et formation, IUFM de Créteil/Paris 6/INRP, pp. 7-33.

NEISSER U., 1976, Cognition and Reality, San Francisco, Freeman.

NGUYEN-XUAN A., GRUMBACH A., 1989, « Modèles informatiques de processus d'acquisition », dans J.-P. Caverni, C. Bastien, P. Mendhelson, G. Tiberghien (eds), Psychologie cognitive : concepts et méthodes, Grenoble, PUG.

NGUYEN-XUAN A., GRUMBACH A., 1984, «Apprendre en résolvant des problèmes : le système humain et les systèmes artificiels », Psychologie Française, $\mathrm{n}^{\circ} 29$ (3/4), pp. 235-242.

OLLIVIER B., 2000, Observer la communication. Naissance d'une interdiscipline, Paris, CNRS éd., coll. CNRS communication.

OPPENHEIM J., 1977, Audiovisuel et formation continue. Étude documentaire. Marché, distribution, pratiques, Paris, INA, Service de la recherche prospective.

PAPADOUDI H., 2000, Technologie et éducation. Contribution à l'analyse des politiques publiques, Paris, PUF.

PAPERT S., 1981, Le jaillissement de l'esprit : ordinateur et apprentissage, Paris, Flammarion.

PAQUETTE G., RICCIARDI-RIGAUT I., de la TEJA C., PAQUIN C., 1997, Le Campus Virtuel à la Télé-Université, Montréal, Télé-Université.

PAQUIN C., PAQUETTE G., BOURDEAU J., RIGAULT C., 1996, Spécification des besoins. Architecture du Campus Virtuel, Rapport interne du projet HyperGuide et Recto, Montréal, Télé-Université, LICEF.

PARAIN C., 1979, Outils, ethnies et développement historique, Paris, éd. sociales. 
PERAYA D., 1999, «Vers les campus virtuels. Principes et fondements techno-sémiopragmatiques des dispositifs de formation virtuels », dans G. Jacquinot-Delaunay et L. Monnoyer, «Le dispositif. Entre usage et concept », Hermès, n 25, pp. 153-168.

PERAYA D., 2002, "Qu'est-ce qu'un campus virtuel ? », dans B. Charlier et D. Peraya, Technologie et innovation en pédagogie, Bruxelles, De Boeck.

PERRIAULT J., 1996, La communication du savoir à distance. Autoroutes de l'information et télésavoirs, Paris, L'Harmattan, coll. Éducation et formation, série Références.

PERRIAULT J., 1989, La logique de l'usage. Essai sur les machines à communiquer, Flammarion.

PERRIAULT J., 1983, «Vingt ans d'EAO : usages, oublis, diversifications », Éducation Permanente, $\mathrm{n}^{\circ} 70-71$, pp. 7-16.

PERIN P., GENSOLLEN M. (eds), 1992, La communication plurielle. L'interaction dans les téléconférences, Paris, CNET/ENST/Documentation française.

PINEAU G., 1985, «L'autoformation dans le cours de la vie : entre l'hétéro et l'écoformation », Éducation Permanente, n 78-79, juin, pp. 25-39.

POINSSAC-NIEL J., 1975, Technologie éducative et histoire, Paris, PUF, coll. Pédagogies d'aujourd'hui.

PORCHER L., 1974, L'école parallèle, Paris, Larousse, coll. Enseignement et pédagogie.

POTEAUX N., 2003, "L'autoformation à l'université : de quelques éléments dialectiques ", dans B. Albero, Autoformation et enseignement supérieur, Paris, Hermès/Lavoisier, pp. 131-140.

POTEAUX N., 2000, "Nouveaux dispositifs, nouvelles dispositions », Les Langues Modernes, $\mathrm{n}^{\circ} 3$, pp. 8-11.

PREVOST H., 1994, L'individualisation de la formation. Autonomie et/ou socialisation, Lyon, Chroniques Sociales.

PRIGOGINE I., STENGERS I., 1981, La nouvelle alliance. Métamorphose de la science, Paris, Gallimard.

PUJOLLE G. et al., 1985, Réseaux et télématique (2 vol.), Paris, Eyrolles.

QUÉAU P., 1986, Éloge de la simulation : de la vie des langages à la synthèse des images, Paris, Champs-Vallon, INA.

QUÉRÉ M., 1994, Vers un enseignement universitaire sur mesure, Paris, Ministère de l'enseignement supérieur et de la recherche, Direction générale des enseignements supérieurs.

QUÉRÉ M. (coord.), 1991, Systèmes experts et enseignement assisté par ordinateur, Paris, Ophrys.

RABARDEL P., 1995, Les hommes et les technologies. Approche cognitive des instruments contemporains, Paris, Armand Colin.

Revue Sciences le l'éducation pour L'Ère nouvelle, 1998, Identité et constitution des Sciences de l'éducation, $\mathrm{n}^{\circ}$ 1-2. 
ROSNAY J. de, 2001, «Biologie et informatique. Promesses et menaces pour le XXIème siècle », dans T. Ferenczi (dir.), Les Défis de la technoscience, Bruxelles, éd. Complexe, coll. Poche, pp. 15-26.

ROSNAY J. de, 1995, L’Homme symbiotique. Regards sur le troisième millénaire, Paris, Seuil.

ROSNAY J. de, 1991, Les rendez-vous du futur, Paris, Fayard.

ROQUEPLO P., 1983, Penser la technique. Pour une démocratie concrète, Paris, Seuil.

RUBENACH J., 1990, De la diapositive au vidéodisque interactif : l'ingénierie des médias an service de l'éducation et de la formation, Thèse de doctorat, Université Paris 13.

SALOMON G., 1981, Communication and Education, Beverly Hills, Sage Publications.

SALOMON G., 1979, Interaction of Media. Cognition and Learning, San Francisco, JosseyBass.

SALOMON J.-J., 1992, Le destin technologique, Paris, Gallimard, coll. Folio actuel, $\mathrm{n}^{\circ} 35$.

SCHAEFFER P., 1980, "Médias de masse : l'école entre Descartes et Mac-Luhan », Perspectives, vol. $10, \mathrm{n}^{\circ} 4$, pp. 465-479.

SCHAEFFER P., 1970, Machines à communiquer (2 t.), Paris, Seuil.

SCHANK R. C., ABELSON R., 1977, Scripts, Plans, Goals and Understanding, Hillsdale, Laurence Erlbaum.

SCHOLER M., 1983, La technologie de l'éducation : concept, bases et applications, Montréal, Presses de l'Université.

SCLOVE R., 1995, 2003, Choix technologiques, choix de société, trad. par I. Jami (Democracy and Technology), Paris, Charles Léopold Mayer/Descartes \& Cie, coll. TechnoCité.

SERRES M. (dir.), 1991, Mission sur l'université à distance. Rapport au Premier ministre, Paris, document multigraphié.

SFEZ L., 2002, Technique et idéologie. Un enjeu de pouvoir, Paris, Seuil, coll. La couleur des idées.

SCHÖN D. A., 1983, The reflective practitioner : How professionals think in action, New York, Basic Books.

SIMON H. A., 1969, 1974, La science des systèmes. Science de l'artificiel, trad. par J.-L. Le Moigne (The Sciences of the Artificial), Paris, Épi.

SIMONDON G., 1989, L'individuation psychique et collective, Paris, Aubier.

SIMONDON G., 1958, 1969, 1989, Du mode d'existence des objets techniques, Paris, Aubier, coll. Philosophie.

SKINNER B. F., 1968, La révolution scientifique de l'enseignement, Bruxelles, Dessart, coll. Psychologie et sciences humaines.

SKINNER B. F., 1958, «Teaching machines », Science, n 128, pp. 69-77.

SKINNER B. F., 1954, "The science of learning and the art of teaching ", Harvard Educational Review, 24, 2, pp. 86-97. 
Savoirs, 5, 2004

SHNEIDERMAN B., 1999, "Human values and the future of Technology : a Declaration of Responsibility ", Computers and Society, Sept., pp. 5-9.

SOUCHON M., POULET S., 1976, Les émissions culturelles à la télévision française : place, programmation, audience, Paris, INA.

STEEPLES C., GOODYEAR P., MELLAR H., 1994, «Flexible learning in higher education : The use of computer-mediated communications ", Computer in Education, vol. 22, n 1-2, pp. 83-90.

STIEGLER B., 1994, 1996, 2001, La technique et le temps. La faute d'Epiméthée (t. 1). La désorientation (t. 2). Le temps du cinéma et la question du mal-être (t. 3), Paris, Galilée.

STIEGLER B., 1997, "Il faut penser les usages », dans Colloque Penser les usages (Arcachon, 27-29 mai 1997), http://www.cent.fr/ust/usages.html

STIEGLER B., 2004a, De la misère symbolique. L'époque byperindustrielle (t. 1), Paris, Galilée.

STIEGLER B., 2004b, Philosopher par accident, Paris, Galilée.

SULTAN J., 2000, "Quelle culture de l'image et des arts visuels pour tous aujourd'hui ?", dans H. Romian (dir.), Pour une culture commune. De la maternelle à l'université, Paris, Hachette Éducation, pp. 333-348.

SULTAN J., VILATTE J.-C. (dir.), 1998, «Ce corps incertain de l'image. Art/Technologie », Champs Visuels, $\mathrm{n}^{\circ} 10$, Juin.

SULTAN J., SATRE J.-P., 1981, La télévision à la porte de l'école : les institutions et la télévision, Paris, La Documentation Française.

TARDIF J., 1998, Intégrer les nouvelles technologies de l'information. Quel cadre pédagogique? Paris, ESF.

TARDY M., 1976, Iconologie et sémiogénèse, Thèse d'État, Université de Strasbourg.

TARDY M., 1974, Le travail indépendant : le centre d'autodocumentation de Marly-le-Roi, Paris, INRP, coll. Recherches pédagogiques, $\mathrm{n}^{\circ} 66$.

TARDY M., 1962, La télévision directe et ses implications pédagogiques, Thèse de troisième cycle, Université de la Sorbonne.

TARDY M., 1966, Le professeur et les images : essai sur l'initiation aux messages visuels, Paris, PUF, coll. Sup/l'Éducateur.

TEILHARD DE CHARDIN P., 1940, 1955, Le Phénomène Humain (t.1), Paris, Seuil.

TEILHARD DE CHARDIN P., 1959, L'avenir de l'homme (t. 5), Paris, Seuil.

THEUREAU J., 1992, Le cours d'action, analyse sémiologique. Essai d'une anthropologie cognitive située, Berne, Peter Lang.

THIBAULT F., 2003, «Coalitions sociales et innovation pédagogique : le cas du Réseau universitaire des centres d'autoformation», dans B. Albero, Autoformation et enseignement supérieur, Paris, Hermès/Lavoisier, pp. 193- 218.

TREMBLAY G., 1990, Les industries de la culture et de la communication au Québec et an Canada, Presses de l'Université du Québec.

TRICOT A., ROUET J. E. (dir.), 1998, «Les hypermédias : approches cognitives et ergonomiques ", Hypertexte et hypermédias, $\mathrm{n}^{\circ}$ hors série.

UTS (Université de tous les savoirs), 2002, Les Technologies, vol. 7, Paris, Odile Jacob coll. Poches, $n^{\circ} 79$. 
VAN DE WIELE T., 1987, «Introduction du vidéodisque en situation scolaire : les travaux de l'Institut National de Recherche Pédagogique», Educational media international, $\mathrm{n}^{\circ} 4$.

VIRILIO P., 1998, La bombe informatique, Paris, Galilée.

WILSON J. R., 1999, "Virtual environments applications and applied ergonomics », Applied Ergonomics, $\mathrm{n}^{\circ} 30$, pp. 3-9.

WILSON J. R., 1997, «Virtual environments and ergonomics : needs and opportunities », Ergonomics, n 40/10, pp. 1057-1077.

WOLTON D., 2000, Internet, et après ? Une théorie critique des nouveaux médias, Paris, Flammarion, coll. Champs, $\mathrm{n}^{\circ} 459$. 